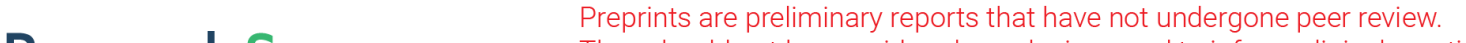 They should not be considered conclusive, used to inform clinical practice, or referenced by the media as validated information. \\ The Effect of Magnetic Field on the Dynamics of Gas Bubbles in Water Electrolysis
}

\section{Yan-Hom Li ( $\nabla$ hom_li@hotmail.com )}

Department of Mechanical and Aerospace Engineering, Chung-Cheng Institute of Technology, National Defense University, Taiwan

\section{Yen-Ju Chen}

Department of Mechanical and Aerospace Engineering, Chung-Cheng Institute of Technology, National Defense University, Taiwan

\section{Research Article}

Keywords: gas bubbles, platinum electrodes, magnetic field result, downward Lorentz forces

Posted Date: March 8th, 2021

DOl: https://doi.org/10.21203/rs.3.rs-283081/v1

License: (1) This work is licensed under a Creative Commons Attribution 4.0 International License. Read Full License

Version of Record: A version of this preprint was published at Scientific Reports on April 30th, 2021. See the published version at https://doi.org/10.1038/s41598-021-87947-9. 


\title{
The effect of magnetic field on the dynamics of gas bubbles in water electrolysis
}

\author{
Yan-Hom Li ${ }^{1,2, *}$, Yen-Ju Chen ${ }^{1, !}$ \\ ${ }^{1}$ Department of Mechanical and Aerospace Engineering, Chung-Cheng Institute of Technology, National \\ Defense University, Taoyuan, 335, Taiwan, \\ ${ }^{2}$ System Engineering and Technology Program, National Yang Ming Chiao Tung University, Hsin-Chu, 300, \\ Taiwan \\ *corresponding. hom_li @ hotmail.com \\ these authors contributed equally to this work
}

\begin{abstract}
In this work, the movement of the gas bubbles evolved from the platinum electrodes in the influence of various magnetic field configurations are experimentally investigated. The oxygen and hydrogen bubbles respectively evolve from the surface of anode and cathode have distinctive behaviors in the presence of magnetic fields due to their paramagnetic and diamagnetic characteristics. The magnetic field perpendicular to the surface of the horizontal electrode induces the revolution of the bubbles. The opposite revolution direction between the oxygen and hydrogen bubbles cause the swirling of the flow and spread out the bubbles between the electrode which enhances the conductivity and electrolysis effectiveness. On the other hand, the vertical electrodes in the influence of a parallel magnetic field induce horizontal Lorentz force which effectively spells out the bubbles and increases the conductivity and electrolysis effectiveness as well. However, when the layouts of the electrode and magnetic field result in upward or downward Lorentz forces which competes with the buoyancy force, the sluggish flow in the duct would hinder the movement of the bubbles and decrease the conductivity and charging performance. This phenomenon affects the corresponding natural convection and mass transport as well. These results propose the optimal layout of the electrode and magnetic field which is useful to enhance the conductivity or the effectiveness in water electrolysis.
\end{abstract}

\section{Introduction}

Gas bubbles are known to affect energy and mass transfer in gas-evolving electrodes. Bubbles that evolve at an electrode may lead to undesired blockage of the electrocatalyst surface ${ }^{1,2}$, and ion-conducting pathways in the electrolyte, resulting in energy losses ${ }^{3}$. Although the associated potential variation for bubble generation is dependent on the operating current density, cell design (electrode geometry and interelectrode instance), and electrolyte composition, several studies have estimated these losses under specific conditions ${ }^{4-6}$. Therefore, how to remove the bubbles from the electrode and reduce the energy losses in the water electrolysis is one of the topics worth being studied further. On the other hand, hydrogen is considered a potential energy carrier, because there is no greenhouse polluting gas. Among methods of hydrogen production, water electrolysis is the most common way because the produced hydrogen has high purity. Therefore, the topics for enhancement of the water electrolysis efficiency have drawn much attention over the past few decades. In recent years, the effects of magnetic fields on hydrogen production have been frequently reported due to certain practical applications. Some studies employed the magnetic field to induce a Lorentz force on the electrolyte, ${ }^{7,8}$ generating convection that enhances mass transfer, and reduces internal resistance and concentration overpotentials. Additionally, convective flow can facilitate the desorption of bubbles and reduce their coverage in the electrodes ${ }^{9-11}$. Reduced overpotentials have been demonstrated for hydrogen evolution reactions by applying magnetic fields perpendicular to the electrodes ${ }^{12}$. The overpotential for hydrogen formation dropped by $10 \%$ when the electrodes were subjected to a magnetic field of 1.5 T. It was found that an external magnetic field has a strong influence on the electrolysis effectiveness. The property of the electrode material can also dominant the efficiency of the electrolysis. A ferromagnetic electrode can give a higher efficiency improvement than a paramagnetic electrode and diamagnetic electrode. The 
effectiveness of hydrogen production is significantly enhanced in a shorter inter-electrode under an upward Lorentz force which is generated by an electromagnetic field ${ }^{8}$. Some studies show that the charging voltage decreases significantly when the distance of the electrode or the concentration of the solution is reduced. The voltage can be further decreased in the presence of a higher magnetic field because of the magnetohydrodynamic effect ${ }^{13,14}$.

On the other hand, many studies have reported that the behaviors of oxygen in water can be affected by an external magnetic field which would enhance the dissolving efficiency of the oxygen or the interface flow velocity 15-21. Most of the results show that when a field strength less than 1T is applied to the solution, the concentration of the dissolved oxygen is increased up to $50 \%$. The paramagnetic property of oxygen molecules makes it possible to control the movement of the oxygen in the solution by an external magnetic field ${ }^{22}$. Oxygen bubbles will move directionally along the surface of the anode when a magnet is faced with the anode. Besides, oxygen bubbles would be in rotational motion in a square electrolyzer, which confines the directional locomotion of oxygen bubbles ${ }^{23}$.

According to the related research mentioned above, bubbles in electrochemical processes are known to induce convection and enhance mass transfer rates and the external magnetic field can improve the electrolysis effectiveness $^{24-28}$. However, we lack a detailed understanding on the intricate dependencies between bubble movement and the magnetic field. Improving the efficiency of existing or emerging electrochemical processes will require a detailed understanding of the behavior of bubbles in electrochemical systems subjected to a magnetic field. In this work, the platinum material is employed as the electrode because of its high electric conductivity, corrosive resistance, and paramagnetic characteristic. Various layouts of the platinum electrodes and magnetic field are designed to investigate the effects of the parallel and the perpendicular field on the movements of the hydrogen and oxygen bubbles during the evolution reaction. The bubbles movement mechanism under the influence of magnetic field is investigated and illustrated, where the conductivity between the electrodes is measured to propose the optimal layouts of the electrodes and magnetic field which can enhance the charging performance or the electrolysis effectiveness.

\section{Materials and method}

\section{Theory}

In water electrolysis, two electrodes are sunk in the electrolysis liquid and there is a certain amount of DC going through them. Water will then be decomposed into hydrogen and oxygen gases. The aqueous solution is electrically conductive due to the existence of electrical ions in the solution, and the electrical conductivity is related to the ion concentration.

In the electrolysis process, the water molecule is the reactant and is dissociated into hydrogen $\left(\mathrm{H}_{2}\right)$ and oxygen $\left(\mathrm{O}_{2}\right)$ under the influence of electricity. Water electrolysis can be classified into four types based on their electrolyte, operating conditions, and ionic agents $\left(\mathrm{OH}^{-}, \mathrm{H}^{+}, \mathrm{O}^{2-}\right)$, however, operating principles and the overall reactions are the same. The four kinds of electrolysis methods are (i) Alkaline water electrolysis (AWE) ${ }^{29-31}$, (ii) Solid oxide electrolysis (SOE) ${ }^{32,33}$ (iii) Microbial electrolysis cells (MEC) $)^{34,35}$. (iv) PEM water electrolysis ${ }^{36,37}$. In pure water electrolysis, the acid-balanced reactions which occur at the anode and cathode surfaces are:

$\mathrm{H}^{+}$reduction at the cathode:

$2 \mathrm{H}^{+}(a q)+2 \mathrm{e}^{-} \rightarrow \mathrm{H}_{2}$

$\mathrm{H}_{2} \mathrm{O}$ oxidation at the anode:

$2 \mathrm{H}_{2} \mathrm{O}(\underline{l}) \rightarrow \mathrm{O}_{2}(g)+4 \mathrm{H}^{+}(a q)+4 \mathrm{e}^{-}$

The overall reaction is as follows:

$2 \mathrm{H}_{2} \mathrm{O} \rightarrow 2 \mathrm{H}_{2}+\mathrm{O}_{2}$

In water electrolysis, gas production is proportional to the electric current. This study will plot the corresponding I-V curves and compare the conductivity of the electrolyte with or without magnetic fields. The conductivity difference (denoted as $\Delta \mathrm{G}$ ) and the conductivity increase rate $\left(\eta_{\mathrm{I}}\right)$ are thus defined as:

$\Delta \mathrm{G}=\mathrm{G}_{\text {magnetic }}-\mathrm{G}_{\text {no magnetic }}$

$\eta_{\mathrm{G}}=\left(\Delta \mathrm{G} / \mathrm{G}_{\mathrm{no} \mathrm{magnetic}}\right) \times 100 \%$ 
where the $G_{\text {magnetic }}$ and $G_{\text {no magnetic }}$ are the conductivity between the electrodes with and without the external field, respectively.

\section{Material preparation}

The electrode material is platinum with an area of $50 \mathrm{~mm} \times 50 \mathrm{~mm}$. Platinum is a kind of transitional metal with paramagnetism and high chemical stability, which is helpful to enhance the movement of the gas bubbles under a magnetic field. The electrolyte is the distilled water without mixing any acid or alkali solution at room temperature. The experiments were carried out in a water tank with dimensions of $400 \mathrm{~mm} \times 240 \mathrm{~mm} \times 200 \mathrm{~mm}$. Two pieces of N35 NdFeB magnet with surface magnetic field strength of $0.22 \mathrm{~T}$ and with dimensions of $100 \mathrm{~mm} \times 50 \mathrm{~mm}$ $\times 10 \mathrm{~mm}$ were placed to generate the magnetic field parallel or perpendicular to the electrodes. Besides, a power supply (GWInstek APS-1102) which can provide the maximum current of 10A is employed to supply a fixed current and measure the charging voltage of the various experimental layouts.

\section{Experimental layout}

The effect of various magnetic field configurations on the behaviors of the gas bubbles was investigated through a systematic experiment. An experimental setup for the oxygen and hydrogen bubbles movement is carried out in the presence of various field configurations as shown in figure 1. Figures $1 \mathrm{a}, 1 \mathrm{~b}$, and $1 \mathrm{c}$ demonstrate the layouts for the horizontal electrodes under the condition of no magnetic and the magnetic field in perpendicular or parallel to the electrodes. Figures $1 \mathrm{~d}, 1 \mathrm{e}$, and $1 \mathrm{f}$ are the layouts for the vertical electrodes under the condition of no magnetic and the magnet in parallel or perpendicular to the electrodes. On the other hand, figures $1 \mathrm{~g}, 1 \mathrm{~h}, 1 \mathrm{i}$ show the layouts for the vertical electrodes with an upward outlet under the condition of no magnetic and the magnet in parallel with or perpendicular to the electrodes to investigate the effect of the vertical force on the conductivity of the system.

\section{Results}

\section{Influence of perpendicular magnetic field}

To understand the effect of a magnetic field that is vertical to the surface of the electrode (denoted as B). The magnets are placed to be parallel and faced with the electrodes as the layouts shown in figures $1 \mathrm{~b}, 1 \mathrm{e}$, and $1 \mathrm{~h}$. Such a perpendicular magnetic field results in a gradient magnetic force (denoted as $F_{\nabla \mathrm{B}}$ ) which is caused by the electrogeneration of paramagnetic molecules in a non-uniform magnetic field and would affect the behaviors of the gas bubbles evolved from a paramagnetic platinum electrode ${ }^{8,27}$. The equation for calculating $F_{\nabla \mathrm{B}}$ is defined as follows $^{38,39}$ :

$\mathrm{F}_{\nabla \mathrm{B}}=\chi_{\mathrm{m}} \mathrm{cB} \nabla \mathrm{B} / \mu_{0}$

Where $\chi_{\mathrm{m}}$ is the molar magnetic susceptibility; $\mathrm{c}$ is the concentration; $\mathrm{B}$ is the magnetic flux density; $\nabla \mathrm{B}$ is the magnetic flux density gradient; $\mu_{0}$ is the magnetic permeability of free space.

\section{Influence of parallel magnetic field}

The influence of the magnetic field parallel to the surface of the electrode is quite different from that perpendicular to the electrode. The layouts for the generation of the magnetic field parallel to the electrode are shown in figures 1c, 1f, 1i. However, it is relatively hard to magnetize the electrode when a magnetic field is parallel to the surface of the electrode. The mechanism affecting the movement of the gas bubbles evolved from the electrodes is the Lorentz force (denoted as $\mathrm{F}_{\mathrm{L}}$ ) which is a force exerted on a charged particle moving in an electricmagnetic field. Only a suitable magnetic field direction can have the Lorentz force, and enhance the convective phenomenon of the MHD in water electrolysis. The magnitude of Lorentz force imposing on the fluid in the duct of MHD configuration is defined as follows ${ }^{40}$ : 
where $\mathrm{F}_{\mathrm{L}}$ is the Lorentz force, $B_{\text {eff }}$ is the effective field which depends on the design parameter of the MHD thruster configuration, $I$ is the electrical current, and $w$ is the distance between the electrodes. The reason for adding a magnetic field in an electric field is to increase the electrolysis rate because charged particles will be forced in the direction perpendicular to the magnetic lines as the magnetic equivalent lines and electrical equivalent lines are orthogonal. A proper layout of magnetic and electric directions will yield a uniform Lorentzinduced flow and improve the electrolysis rate.

In general, if the magnetic field is uniform, $F_{\nabla B}$ can be neglected because its value is much lower than $F_{L}$ when the parallel magnetic field is applied ${ }^{41}$. Therefore, the effects of magnetic fields on the behaviors of gas bubbles are mainly induced by $\mathrm{F}_{\mathrm{L}}$. However, for a non-uniform magnetic field, after the application of a perpendicular magnetic field, $F_{L}$ is much lower than $F_{\nabla B}{ }^{38,42}$. For the different scenarios, the magnetic field effects on the behaviors may be mainly either caused by $F_{L}$ or $F_{\nabla B}$. Thus, it is possible to distinguish the effects caused by $F_{L}$ from those by $\mathrm{F}_{\nabla \mathrm{B}}$.

\section{Effects of magnetic field configurations on the gas bubbles evolved from horizontal electrodes}

Figure 2 a shows that the charging voltage linearly enhances with the increasing current density for the platinum electrodes placed horizontally under various field layouts, as shown in figures $1 \mathrm{a}, 1 \mathrm{~b}$, and $1 \mathrm{c}$. Figure $2 \mathrm{~b}$ shows the effect of the magnetic field on the conductivity between the two platinum electrodes, where the applied magnetic field is found to effectively reduce the charging voltage when the electrodes are faced with the magnets or perpendicular to the magnets. This result means both the parallel and perpendicular magnetic fields would enhance the conductivity between the electrodes. The perpendicular magnetic field generated by the parallel placed magnets has a more significant influence on the enhancement of the conductivity for the horizontally placed electrodes. As shown in figure $2 \mathrm{a}$, at the same current density of $200 \mathrm{~mA} / \mathrm{cm}^{2}$, generated by an electrical current of $5 \mathrm{~A}$, the horizontal electrodes faced with parallel magnets show a lower charging voltage of $11.3 \mathrm{~V}$, which response to a higher conductivity of $\mathrm{G}=0.443$ shown in figure $2 \mathrm{~b}$, and the increasing ratio $\eta_{\mathrm{G}}$ is about $13.6 \%$ compared to the one without magnetic field. Figure 3 demonstrated the sequential movement of the gas bubbles in the duct between the two horizontal electrodes subjected to the perpendicular magnetic field. The circular movements are found for both the oxygen and hydrogen bubbles. The paramagnetic oxygen bubbles spread away from the anode surface (lower electrodes) and make a counterclockwise revolution when the N-pole of the magnet faces with the anode, then the upward buoyancy force results in the swirling up of the oxygen bubbles. On other hand, the cathode (upper electrode) faces with $\mathrm{S}$-pole of the magnet produces hydrogen bubbles which nearly adhere to the cathode surface due to the influence of the buoyancy force and rotate slowly in the clockwise direction from the top view observation. It is seen that the revolution direction of hydrogen bubbles is opposite to the direction of the oxygen bubbles due to the diamagnetic characteristic of the hydrogen molecular. Additionally, both the revolution direction of the oxygen and hydrogen bubbles reverse when the pole of the magnet faced with the anode and cathode electrode changes to $\mathrm{S}$ and $\mathrm{N}$ respectively. The different revolution directions between the upper and lower bubbles lead to the twist of the flow between the electrodes, which serves as a stirrer to increase hydrodynamics. This phenomenon indicates that the horizontal rotation movement driven by the interaction between the paramagnetic/diamagnetic bubbles and the magnetic field is helpful to make oxygen and hydrogen bubbles detached from the surface of the electrodes and reduce the internal resistance.

When the parallel magnetic field produced by the vertically placed magnets impose on the horizontal electrodes, the movement of the gas bubbles are quite different from those shown in figure 3 . The locomotion of the gas bubbles is mainly caused by the horizontal Lorentz force formed by the orthogonal layout of the electrical and magnetic field. Base on the field configuration shown in figure 4, the direction of Lorentz force is into the paper. The oxygen bubble spread away from the surface of the anode when the concentration of oxygen bubbles near the surface is saturated. The Lorentz-force-induced flow rapidly flushes out the oxygen bubbles in the inward direction. Thus, the oxygen bubble will not adhere to the surface of the anode or stay in the duct for a long time, which reduces the internal resistance and charging voltage of such an MHD configuration. However, according to equation (3), the number of hydrogen bubbles evolved from the cathode should be two times of the oxygen from the anode and mostly gather on the cathode surface due to the upward buoyancy force. This result hurdles the movement of the hydrogen bubbles and impacts the enhancement of the overall conductivity of the fluid in the 
duct. This phenomenon can be used to explain the lower conductivity of $\mathrm{G}=0.42$ compared to the parallel magnets as shown in figure $2 \mathrm{~b}$ under an identical electrical current of $5 \mathrm{~A}$.

On the other hand, if the hydrogen bubbles were evolved from the lower electrode as shown in figure 5a, the bubbles distribution pattern varies significantly compared to figure 4a. Figure 5a shows that the gas bubbles in the dust become denser than that shown in figure $4 \mathrm{a}$ and almost scatter in the whole dust within a show period. This is because the weight of the hydrogen bubbles is lighter and the amount of the hydrogen bubbles evolved from the cathode electrode is more than the oxygen bubbles. When the concentration of hydrogen bubbles near the surface is saturated, the speed of hydrogen bubbles spread away from the surface of the cathode is much faster due to the effect of the upward buoyancy force. However, the inward Lorentz force does not stronger enough to spell out the bubbles in the dust toward the exit. As a result, the internal resistance of the electrodemagnet layout shown in figure $5 \mathrm{a}$ is larger than the one shown in figure $4 \mathrm{a}$. The conductivity of $\mathrm{G}=0.406$ for the cathode at the lower position is smaller than $\mathrm{G}=0.42$ for the anode at the lower position under an identical electrical current of $5 \mathrm{~A}$.

\section{Effects of magnetic field configurations on the gas bubbles evolved from vertical electrodes}

To eliminate the effect of the buoyancy force on the stagnation of the bubbles on the upper electrode, the experiments for the layout of vertical electrodes in the presence of various magnetic field configurations, as shown in figures $1 \mathrm{~d}, 1 \mathrm{e}$, and 1f are carried out. Figure $5 \mathrm{~b}$ demonstrates the movement of the gas bubbles for the layout shown in figure $1 \mathrm{f}$, which are turned $90^{\circ}$ clockwise from the case shown in figure 5a. The overall gas bubbles in the duct decrease significantly, compared to figure 5a. When the concentration of oxygen and hydrogen bubbles respective near the anode (right) and the cathode (left) surface is saturated, the inward Lorentz force rapidly flushes the bubbles out of the exit. The effect of the buoyancy force is still seen from the time of $t=1.5 \mathrm{~s}$ to $\mathrm{t}=2.4 \mathrm{~s}$ near the corner between the cathode and magnet S-pole when the amount of the hydrogen bubbles is increased gradually. Under the layout shown in figure $5 b$, the horizontal Lorentz force has a more prominent influence than the upward buoyancy force. Thus, the overall bubbles stay in the dust reduced significantly and lead to a better conductivity as shown in figure 6.

Figure 6a shows that the charging voltage linearly enhances with the increasing current density for the platinum electrodes placed vertically in the presence of various field conditions, as shown in figures $1 \mathrm{~d}, 1 \mathrm{e}$, and 1f. Under this electrode-magnet layout, at the same current density of $200 \mathrm{~mA} / \mathrm{cm}^{2}$, which is generated by an electrical current of $5 \mathrm{~A}$, the vertical electrodes subjected to the parallel magnets shows the lowest charging voltage of $11.1 \mathrm{~V}$, which responding to the conductivity of $\mathrm{G}=0.45$ shown in figure $6 \mathrm{~b}$, and the increasing ratio

$\eta_{\mathrm{G}}$ is about $4.7 \%$ compared to the one without a magnetic field, and is better than the conductivity of $\mathrm{G}=0.42$ and $\mathrm{G}=0.40$ for the layouts shown in figure $4 \mathrm{a}$ and figure $5 \mathrm{a}$, respectively.

Additionally, the layout of the vertical electrodes with horizontal magnets shown in figure $5 \mathrm{~b}$ has nearly the same conductive of $\mathrm{G}=0.45$ with $\mathrm{G}=0.443$ for the configuration of horizontal electrodes faced with horizontal magnets as shown in figure 3. However, when the vertical electrodes are faced with the vertical magnets, the charging voltage is increased from $11.1 \mathrm{~V}$ to $12.5 \mathrm{~V}$ and the conductivity decreases from 0.45 to 0.4 , which is even lower than $\mathrm{G}=0.431$ for the electrodes without a magnetic field, under an electrical current of $5 \mathrm{~A}$. This result reveals that the vertical rotation movement of the bubbles driven by the interaction between the paramagnetic/diamagnetic bubbles and the magnetic field is not helpful to make oxygen and hydrogen bubbles detached from the surface of the electrodes.

\section{Effect of vertical Lorentz force on the movement of the bubbles}

It is known that different magnetic directions will result in upward or downward Lorentz forces, which will affect the corresponding natural convection and mass transport as well. The experiments for the layouts shown in figures $1 \mathrm{~g}, 1 \mathrm{~h}$, and $1 \mathrm{i}$ are carried out to investigate the effect of the vertical Lorentz force and the vertical revolution of the bubbles on the conductivity of the fluid in the duct. Figure 7 shows the movement of the bubbles for the layout of the vertical electrodes perpendicular to the vertical magnets which form a vertical Lorentz force. The sequential images in figure 7 show that the oxygen bubbles move rightward and perpendicular to the surface of the anode when the concentration of oxygen bubbles near the anode surface is saturated. The cluster of oxygen bubbles near the N-pole of the magnet is repelled from the surface of the magnet while the bubbles are attracted to the S-pole of the magnet. This result is caused by the paramagnetic characteristic of the oxygen bubbles, which are magnetized by the external magnetic field and weakly repelled by the magnet N-pole and attracted by the S-pole as shown in the schematic diagram in figure $7 \mathrm{~b}$. The results shown in figure $7 \mathrm{a}$ also demonstrates the influence of 
the downward Lorentz force $\left(\mathrm{F}_{\mathrm{L}}\right)$ on the movement of the bubbles. Numerous reports have shown $\mathrm{F}_{\mathrm{L}}$ produced by the charges moving under the applied magnetic field induces an electrolyte convection ${ }^{43-47}$. It is generally accepted that the enhancement of electrochemical reactions can be attributed to mass transport which is enhanced by $\mathrm{F}_{\mathrm{L}}{ }^{43-}$

${ }^{50}$. However, the direction of the imposed $F_{L}$ plays an important role to determine the efficiency of the reaction to be increased or decreased. The downward $\mathrm{F}_{\mathrm{L}}$ will compete with the upward buoyancy force, which results in the slower movement of oxygen bubbles when the bubbles spread away from the anode surface due to the saturated concentration. Furthermore, the repulsion of the oxygen bubbles by the N-pole magnet makes a curve moving path and increases the moving distance of the bubbles. As a result, the oxygen bubbles move slowly and stay longer in the duct of a layout influenced by downward Lorentz force. This type of layout increases the internal resistance and needs a higher charging voltage than the vertical electrodes without applying a magnetic field as shown in figure $1 \mathrm{~g}$.

The results shown in Figure 8a demonstrate the quantitative comparison for the layouts with an upward exit in the presence of various field configurations. Figure 8 shows that the external magnetic field have a negative impact on the conductivity between the electrodes based on the layout of figure $1 \mathrm{~h}$. At the same current density of $200 \mathrm{~mA} / \mathrm{cm}^{2}$, which is generated by an electrical current of $5 \mathrm{~A}$, the vertical electrodes subjected to a downward Lorentz force shows a higher charging voltage of $12.8 \mathrm{~V}$, which respond to a lower conductivity of $\mathrm{G}=0.39$ shown in figure $8 \mathrm{~b}$, and the increasing ratio $\eta_{\mathrm{G}}$ is about $-6.25 \%$ compared to the one without magnetic field. The charging voltage reduces slightly when the upward Lorentz force is imposed on the bubbles. The upward Lorentz is helpful to overcome the weight of the static fluid in the duct and generate the Lorentz-force-induced flow. However, the closed bottom of the duct fails to provide sufficient inlet flow to continually generate significant Lorentz force. Furthermore, the repulsion of the oxygen bubbles by the N-pole magnet increases the moving path of the bubbles and prolongs the staying period in the duct. As a result, the upward Lorentz force does not have an effectiveness to reduce the charging voltage of the layout though the upward buoyancy force is imposed. The conductivity is slightly increased to $\mathrm{G}=0.39$ to 0.396 , still lower than $\mathrm{G}=0.417$ for the layout without a magnetic field. On the other hand, when the vertical magnets are placed to face with the vertical electrodes under an upward-exit layout, the charging voltage is higher than the electrodes without applying a magnetic field as well. As shown in figure $8 \mathrm{a}$, when the vertical electrodes are faced with the vertical magnets, the charging voltage is increased from $12 \mathrm{~V}$ to $12.3 \mathrm{~V}$, and the conductivity decreases from $\mathrm{G}=0.417$ to $\mathrm{G}=0.407$. Those results indicate that neither the vertical revolution of the bubbles nor the vertical Lorentz is helpful to reduce the internal resistance in the duct of top-exit layout, which means the external magnetic field is not useful for the enhancement of the conductivity of configuration shown in figure $1 \mathrm{~g}$.

\section{Discussion}

The magnetic field perpendicular to the surface of the electrode would induce the revolution of the bubbles. For the horizontal electrodes, the oxygen and hydrogen bubbles respectively evolved from anode and cathode rotate in the opposite direction leads to the swirling flow between the electrode and spread out the bubbles from the duct. Thus, the internal resistance is reduced and the conductivity and water electrolysis effectiveness are increased. On the other hand, for the vertical electrodes, the horizontal Lorentz force induced by the magnetic field parallel to the electrode dominates the movement of the bubbles and enhances the conductive significantly. However, the vertical electrodes faced with a vertical magnet that induces vertical rotation movement of the bubbles is not helpful to make bubbles detached from the surface of the electrodes. For the layout of the vertical electrodes with an upward exit, neither the perpendicular nor the parallel magnetic field is in favor of the movement of the bubbles. Those experimental results propose the optimal layouts of the electrodes and magnetic field to enhance the conductivity or the effectiveness for various applications which involve water electrolysis.

\section{Data availability}

The datasets generated during the current study are available from the corresponding author on reasonable request.

\section{References}

1. Thorne, R.J., Sommerseth, C., Ratvik, A.P., Rørvik, S., Sandnes, E., Lossius, L.P., Linga, H., \& Svensson, A.M. Bubble evolution and anode surface properties in aluminium electrolysis. J. Electrochem. Soc. 162, E104-E114 (2015) 
2. Vogt, H. The quantities affecting the bubble coverage of gas-evolving electrodes. Electrochim. Acta $\mathbf{2 3 5}$, 495499 (2017)

3. Kiros, Y., \& Martin, B. Low energy consumption in chlor-alkali cells using oxygen reduction electrodes. Int. J. Electrochem. Sci. 3, 444-451. (2008)

4. Cooksey, M.A., Taylor, M.P., \& Chen, J.J.J. Resistance due to gas bubbles in aluminum reduction cells. JOM 60, 51-57 (2008)

5. Haupin, W.E. A scanning reference electrode for voltage contours in aluminum smelting cells. JOM 23, 46-49 (1971)

6. Wang, Y.-1., Tie, J., Tu, G., Sun, S., Zhao, R., \& Zhang, Z. Effect of gas bubble on cell voltage oscillations based on equivalent circuit simulation in aluminum electrolysis cell. Trans. Nonferrous Met. Soc. China 25, 335-344 (2015)

7. Matsushima, H., Kiuchi, D., \& Fukunaka, Y. Measurement of dissolved hydrogen supersaturation during water electrolysis in a magnetic field. Electrochim. Acta 54, 5858-5862 (2009)

8. Lin, M.Y., Hourng, L.W., and Kuo, C.W. The effect of magnetic on hydrogen production efficiency in water electrolysis. Int. J. Hydrogen Energy 37, 1311-1320 (2012)

9. Iida, T., Matsushima, H., \& Fukunaka, Y. Water electrolysis under a magnetic field. J. Electrochem. Soc. 154, E112-E115 (2007)

10. Koza, J.A., Mu“ hlenhoff, S., Z_abin_ski, P., Nikrityuk, P.A., Eckert, K., Uhlemann, M., Gebert, A., Weier, T., Schultz, L., \& Odenbach, S. Hydrogen evolution under the influence of a magnetic field. Electrochim. Acta 56, 2665-2675 (2011)

11. Matsushima, H., Iida, T., and Fukunaka, Y. Gas bubble evolution on transparent electrode during water electrolysis in a magnetic field. Electrochim. Acta 100, 261-264 (2013)

12. Diao, Z., Dunne, P.A., Zangari, G., \& Coey, J.M.D. Electrochemical noise analysis of the effects of a magnetic field on cathodic hydrogen evolution. Electrochem. Commun. 11, 740-743 (2009)

13 Sueptitz, R., Tschulik,K., Uhlemann, M., Gebert, A., \& Schultz, L. Impact of magnetic field gradients on the free corrosion of iron. Electrochim. Acta 55, 5200-5203 (2010).

14 Rhen, F.M.F., Hinds, G., and Coey, J.M.D. Magnetic field effect on the rest potential of zinc. Electrochem. Commun. 6, 413-416 (2004)

15. Liu, B. N., Efimov, M. L., Kul'Sartov, V. K., \& Iakupova, R. M. Movement of dissolved oxygen in a constant magnetic field. Biofizika 23, 159-161 (1978)

16. Boulware, J. C., Ban, H., Jensen, S., \& Wassom, S. Modeling of the dynamics of a slug of liquid oxygen in a magnetic field and experimental verification. Cryogenics 50, 401-409 (2010)

19. Ueno, S., \& Harada, K. Redistribution of dissolved oxygen concentration under strong DC magnetic fields. IEEE Trans. Magn. 18, 1704-1706 (1982)

17. Hirota, N., Ikezoe, Y., Uetake, H., Nakagawa, J., \& Kitazawa, K. Magnetic field effect on the kinetics of oxygen dissolution into water. Mater. Trans. JIM 41, 976-980 (2000)

18. Bao, S. R., Zhang, R. P., Zhang, Y. F., Tang, Y., Zhang, J. H., \& Qiu, L. M.. Enhancing the convective heat transfer in liquid oxygen using alternating magnetic fields. Appl. Therm. Eng. 100, 125-132 (2016)

15. Bao, S. R., Zhang, R. P., Wang, K., Zhi, X. Q., \& Qiu, L. M. Free-surface flow of liquid oxygen under nonuniform magnetic field. Cryogenics 81, 76-82. (2017)

21 Pouryazdanpanah K. E., \& Chen, Y. Study of Magnetic Field Effects on the Oxygen Transfer in Liquid Lead Cavity Flow Using the Lattice Boltzmann Method. J. Heat Transf. 142 (2020)

22. Takamizawa, S., Nakata, E. I., \& Akatsuka, T. Magnetic Behavior of a 1D Molecular - Oxygen System Included within a Transformable Single - Crystal Adsorbent. Angew. Chem.-Int. Edit 45, 2216-2221 (2006)

23. Wang, K., Pei, P., Pei, Y., Ma, Z., Xu, H., \& Chen, D. Magnetic field induced motion behavior of gas bubbles in liquid. Sci. Rep. 6, 1-6. (2016)

24. Vogt, H. Superposition of microconvective and macroconvective mass transfer at gas-evolving electrodes $-\mathrm{a}$ theoretical attempt. Electrochim. Acta 32, 633-636. (1987)

25. Vogt, H., \& Stephan, K. Local microprocesses at gas-evolving electrodes and their influence on mass transfer. Electrochim. Acta 155, 348-356. (2015)

26. Dees, D.W., \& Tobias, C.W. Mass transfer at gas evolving surfaces. J. Electrochem. Soc. 134, 1702-1713 (1987) 
27. Yang, X., Karnbach, F., Uhlemann, M. Odenbach, S., and Eckert, K. Dynamics of single hydrogen bubbles at a platinum microelectrode. Langmuir 31, 8184-8193 (2015)

28. Angulo, A., van der Linde, P., Gardeniers, H., Modestino, M., \& Rivas, D. F. Influence of bubbles on the energy conversion efficiency of electrochemical reactors. Joule, 4, 555-579. (2020)

29. Zeng, K., \& Zhang, D., Recent progress in alkaline water electrolysis for hydrogen production and applications, Prog. Energy Combust. Sci. 36, 307-326 (2010)

30. Kumar, S. S., Ramakrishna, S. U. B., Krishna, S. V., Srilatha, K., Devi, B. R., \& Himabindu, V. Synthesis of titanium (IV) oxide composite membrane for hydrogen production through alkaline water electrolysis. South African J. Chem. Eng, 25, 54-61 (2018)

31. Kumar, S. S., Ramakrishna, S.U.B., Reddy, D. S., Bhagawan, D., \& Himabindu, V. Synthesis of Polysulfone and zirconium oxide coated asbestos composite separators for alkaline water electrolysis, Int. J. Chem. Eng. Process Technol. 3, 1035/1-6. (2017)

32. Ni, M., Leung, M.K.H., \& Leung, D.Y.C. Technological development of hydrogen production by solid oxide electrolyzer cell (SOEC), Int. J. Hydrogen Energy 33, 2337-2354 (2008)

33. Laguna-Bercero, M.A. Recent advances in high temperature electrolysis using solid oxide fuel cells: a review, J. Power Sources 203, 4-16 (2012)

34. Kadier, A., Simayi, Y., Abdeshahian, P., Azman, N. F., Chandrasekhar, K., \& Kalil, M. S. A comprehensive review of microbial electrolysis cells (MEC) reactor designs and configurations for sustainable hydrogen gas production. Alex. Eng. J. 55, 427-443 (2016)

35.Kadier, A., Kalil, M. S., Abdeshahian, P., Chandrasekhar, K., Mohamed, A., Azman, N. F., Logroño, W., Simayi, Y. \& Hamid, A. A. Recent advances and emerging challenges in microbial electrolysis cells (MECs) for microbial production of hydrogen and value-added chemicals. Renew. Sust. Energ. Rev., 61, 501-525 (2016)

36. Sapountzi, F. M., Gracia, J. M., Fredriksson, H. O., \& Niemantsverdriet, J. H. Electrocatalysts for the generation of hydrogen, oxygen and synthesis gas. Prog. Energy Combust. Sci., 58, 1-35 (2017)

37. Aricò, A. S., Siracusano, S., Briguglio, N., Baglio, V., Di Blasi, A., \& Antonucci, V. Polymer electrolyte membrane water electrolysis: status of technologies and potential applications in combination with renewable power sources. J. Appl. Electrochem. 43, 107-118. (2013)

38. Ragsdale, S. R., Grant, K. M., \& White, H. S. Electrochemically generated magnetic forces. Enhanced transport of a paramagnetic redox species in large, nonuniform magnetic fields. J. Am. Chem. soc. 120, 1346113468 (1998)

39. Gorobets, O. Y., Gorobets, V. Y., Derecha, D. O., \& Brukva, O. M. Nickel electrodeposition under influence of constant homogeneous and high-gradient magnetic field. J. Phys. Chem. 112, 3373-3375 (2008)

40. Cébron, D., Viroulet, S., Vidal, J., Masson, J. P., \& Viroulet, P. Experimental and theoretical study of magnetohydrodynamic ship models. PloS one, 12, e0178599. (2017)

41. Rhen, F. M., Hinds, G., \& Coey, J. M. D. Magnetic field effect on the rest potential of zinc. Electrochem. Commun., 6, 413-416 (2004)

42. Lioubashevski, O., Katz, E., \& Willner, I. Effects of magnetic field directed orthogonally to surfaces on electrochemical processes. J. Phys. Chem. C. 111, 6024-6032 (2007)

43. Lu, Z., Huang, C., Huang, D., \& Yang, W. Effects of a magnetic field on the anodic dissolution, passivation and transpassivation behaviour of iron in weakly alkaline solutions with or without halides. Corros. Sci. 48, 3049-3077 (2006).

44. Rhen, F. M. F., \& Coey, J. M. D. Magnetic field induced modulation of anodic area: Rest potential analysis of Zn and Fe. J. Phys. Chem. C. 111, 3412-3416 (2007)

45. Koza, J. A., Karnbach, F., Uhlemann, M., McCord, J., Mickel, C., Gebert, A., ... \& Schultz, L..

Electrocrystallisation of $\mathrm{CoFe}$ alloys under the influence of external homogeneous magnetic fields-Properties of deposited thin films. Electrochim. Acta 55, 819-831 (2010)

46. Yang, X., Eckert, K., \& Odenbach, S. Oscillatory Lorentz-force-driven flows during potentiostatic current oscillations in magnetic fields. Electrochem. Commun. 12, 1576-1580 (2010)

47. Sueptitz, R., Tschulik, K., Uhlemann, M., Schultz, L., \& Gebert, A.. Magnetic field effects on the active dissolution of iron. Electrochim. Acta 56, 5866-5871 (2011) 
48. Chouchane, S., Levesque, A., Zabinski, P., Rehamnia, R., \& Chopart, J. P. Electrochemical corrosion behavior in $\mathrm{NaCl}$ medium of zinc-nickel alloys electrodeposited under applied magnetic field. J. Alloys Compd. 506, 575-580 (2010)

49. Yang, X., Chen, S., Wang, C., \& Liang, L. I. Application of in situ digital holography to the study of the effect of a magnetic field on the anodic dissolution of iron in thichloroacetic acid. J. Serb. Chem. Soc. 71, 6773 (2006)

50. Mühlenhoff, S., Mutschke, G., Koschichow, D., Yang, X., Bund, A., Fröhlich, J., \& Eckert, K. Lorentzforce-driven convection during copper magnetoelectrolysis in the presence of a supporting buoyancy force. Electrochim. Acta 69, 209-219 (2012)

\section{Competing interests}

The authors declare no competing interests.

\section{Acknowledgements}

This study was funded by the Ministry of Science and Technology of the Republic of China (Taiwan) through Grant MOST 109-2221-E-606-003.

\section{Author contributions}

Y.H. conceived the study and conducted the data analysis. Y.J. implemented the experiments and plotted the diagrams. Y.H. wrote the paper, with contributions from all authors. All authors reviewed the manuscript.

\section{Additional information}

To see the movies for experimental results demonstrated in the manuscript please find the below Google drive link https://drive.google.com/drive/folders/1stiJt7hFgdK9YdYSq42i4MtG81vP4a30 
a

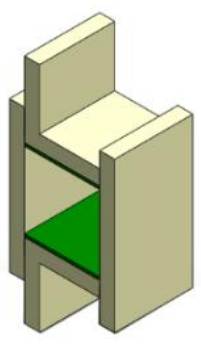

d

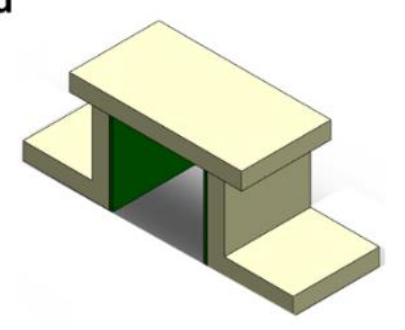

g

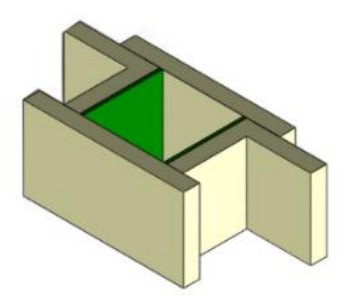

b

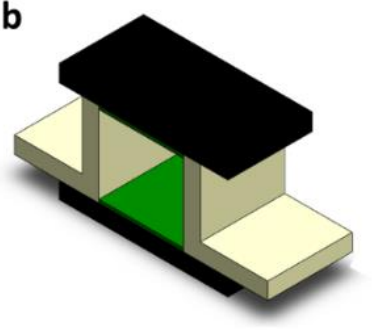

e

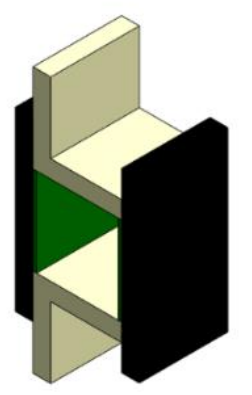

C

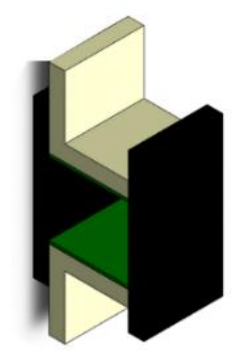

f

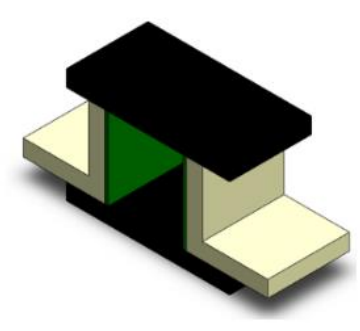

i

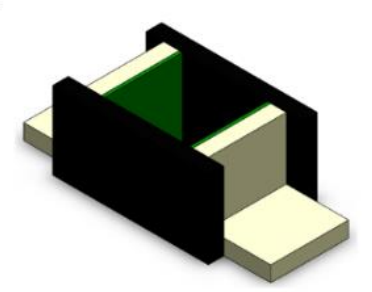

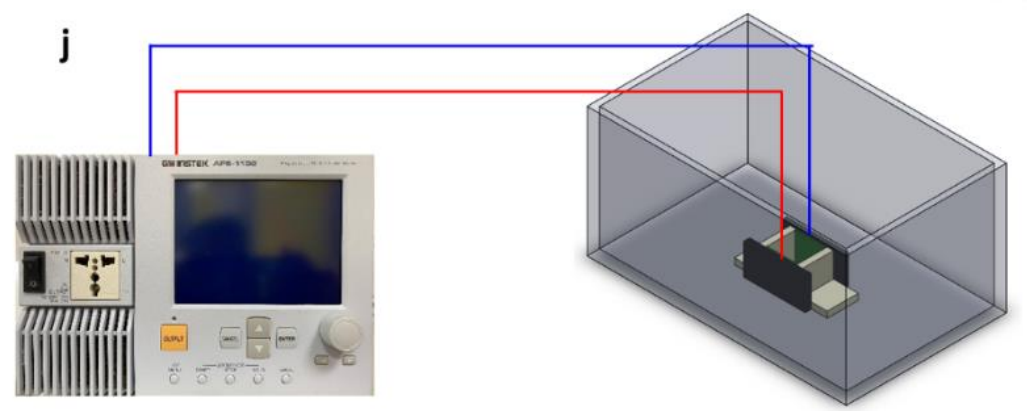

Figure 1. A schematic diagram of the experimental setup and the layout of the electrodes and magnetic field. The black area is the pair of N35 magnets (100 mm long, 50mm wide, and 10mm high) whose surface field strength is nearly $0.22 \mathrm{~T}$, the green part is the electrodes (50 $\mathrm{mm}$ long and $50 \mathrm{~mm}$ wide), the yellow part is the isolating plastic that is used to form the duct. Various magnetic field configurations were designed to investigate the effect of the field on the behaviors of the gas bubbles. (a) The electrodes were placed horizontally without magnets. (b) The electrodes were placed horizontally and faced with two horizontal magnets. (c) The electrodes were placed horizontally and perpendicular to the magnets which forms the Lorentz force (denoted as $F_{L}$ ) in a horizontal direction. (d) The electrodes were placed vertically without magnet. (e) The electrodes were placed vertically and faced with vertical magnets. (f) The electrodes were placed vertically and perpendicular to magnets which forms the Lorentz force in horizontal direction. $(\mathrm{g})$ The electrodes were placed vertically without a magnet and with an upward exit. (h) The electrodes were placed vertically and faced with vertical magnets and with an upward exit. (i) The electrodes were placed vertically and perpendicular to magnets which forms the Lorentz force in a vertical direction and with an upward exit. (j) All the experiments were conducted in a $40 \mathrm{~cm}$ long, $24 \mathrm{~cm}$ wide, and $20 \mathrm{~cm}$ high water tank. The electrodes were connected to the power supply (GWInstek APS-1102) which can provide the maximum current of 10A. 

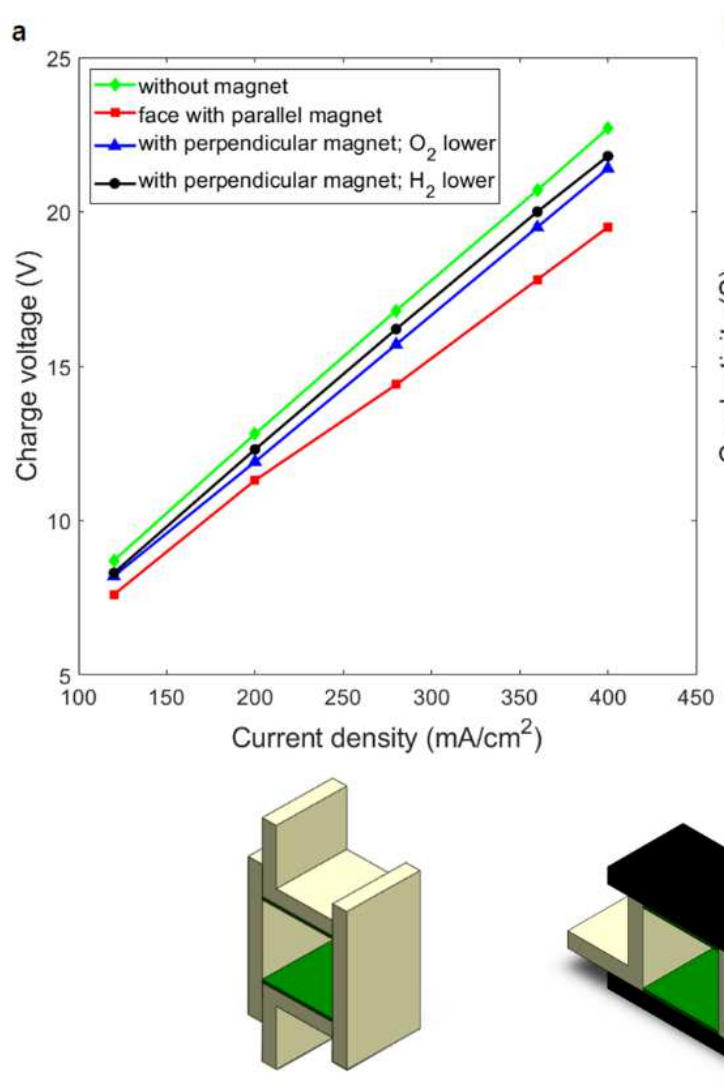

without magnet
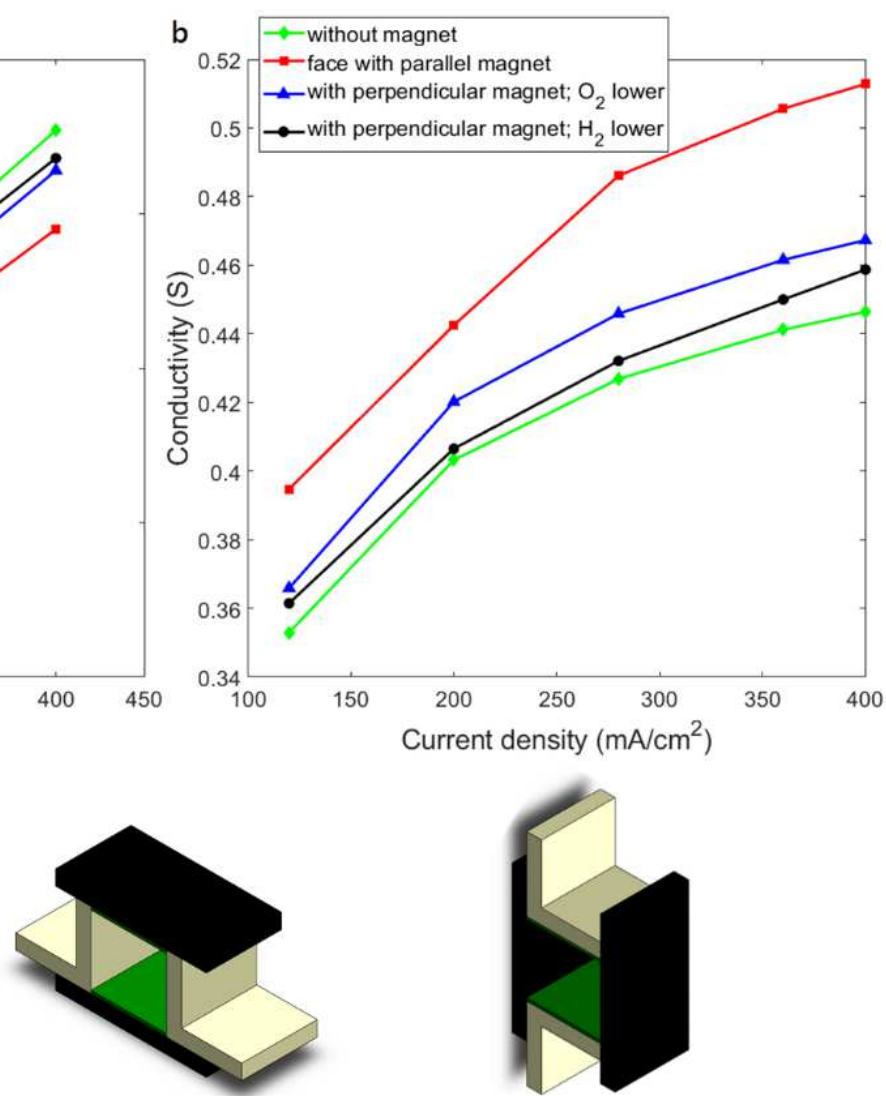

face with parallel magnet

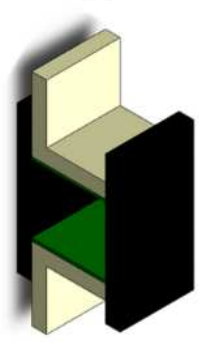

with perpendicular magnet

Figure 2. Comparison of the conductivity for horizontal platinum electrodes under the effects of various magnetic field configurations. (a) Charging voltage vs. current density for the experimental layouts shown in figures 1a, 1b, and 1c. (b) Conductivity vs. current density for the experimental layouts shown in figures 1a, 1b, and $1 \mathrm{c}$. 


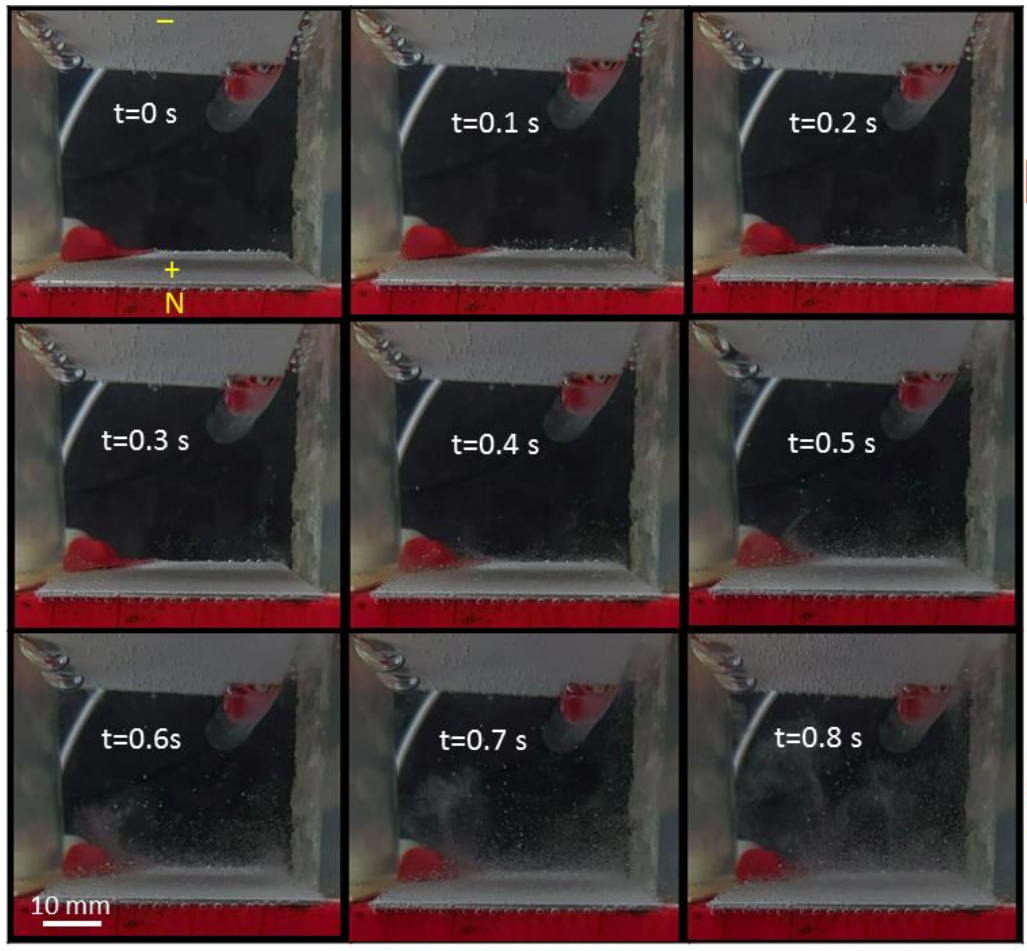

a

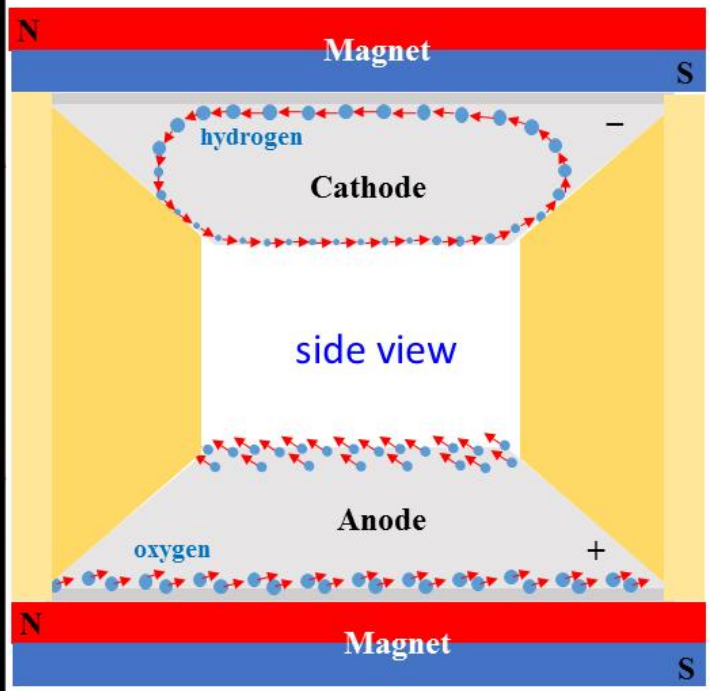

b

Figure 3. Swirling gas bubbles under the effect of the magnetic field perpendicular to the electrode surface. (a) Sequential images of the locomotion of the oxygen (lower electrode) and hydrogen (upper electrode) on the platinum electrodes subjected to a perpendicular magnetic field. The experimental layout is shown in figure 1(b) with an electrical current of $5 \mathrm{~A}$. (b) The schematic diagram for the revolution of the oxygen and hydrogen bubbles.

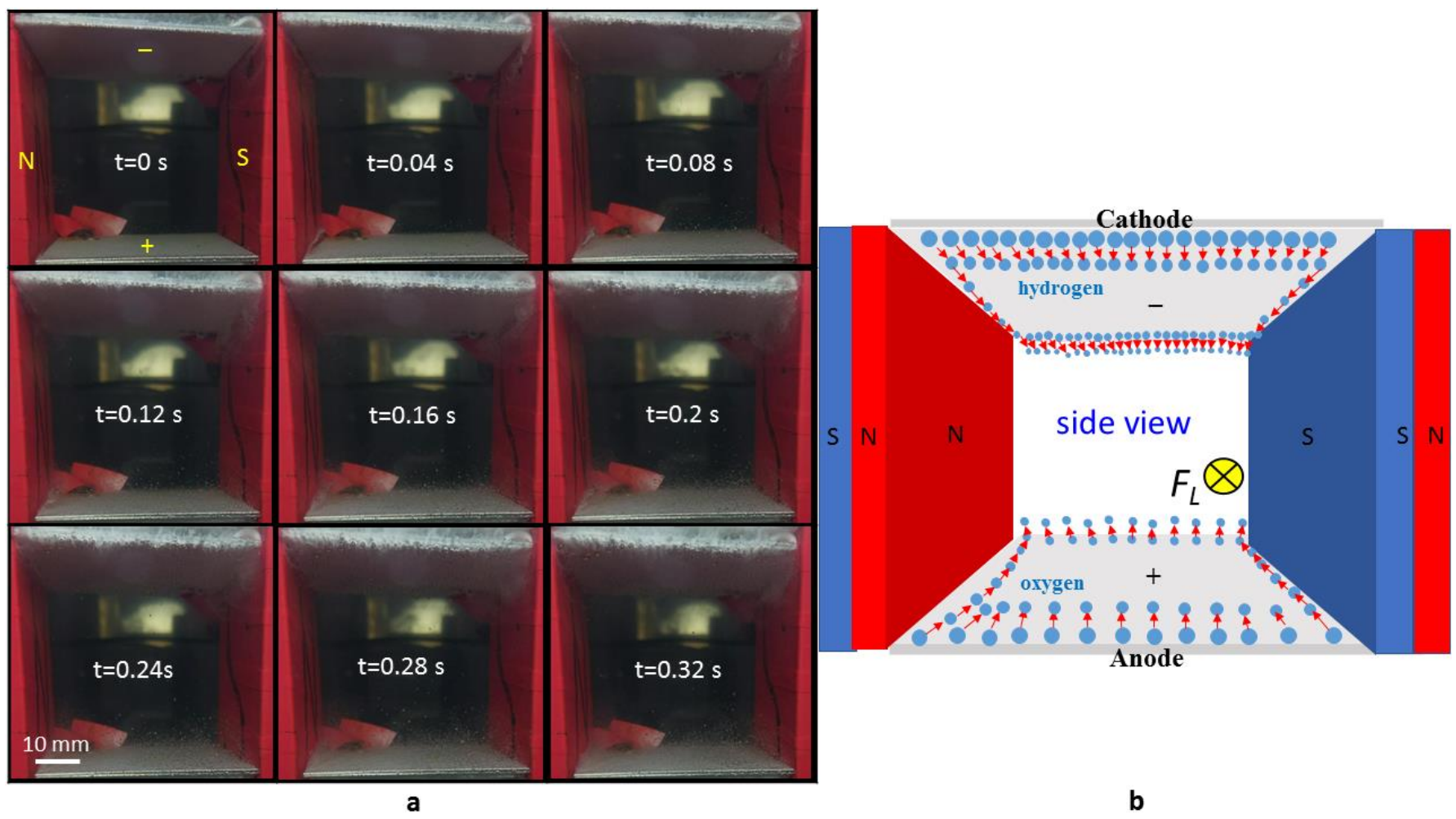

Figure 4. Locomotion of gas bubbles produced by horizontal platinum electrodes under the effect of horizontal Lorentz force generated by a horizontal magnetic field and a perpendicular electric field. 
(a)Sequential images of the oxygen and hydrogen bubbles detached from the lower and upper platinum electrode, respectively. The experimental layout is shown in figure 1(c) under the electrical current of $5 \mathrm{~A}$. The $\mathrm{N}$-pole and S-pole of the magnet are at the left and right sides, respectively. (b) The schematic diagram for the locomotion of the gas bubbles.

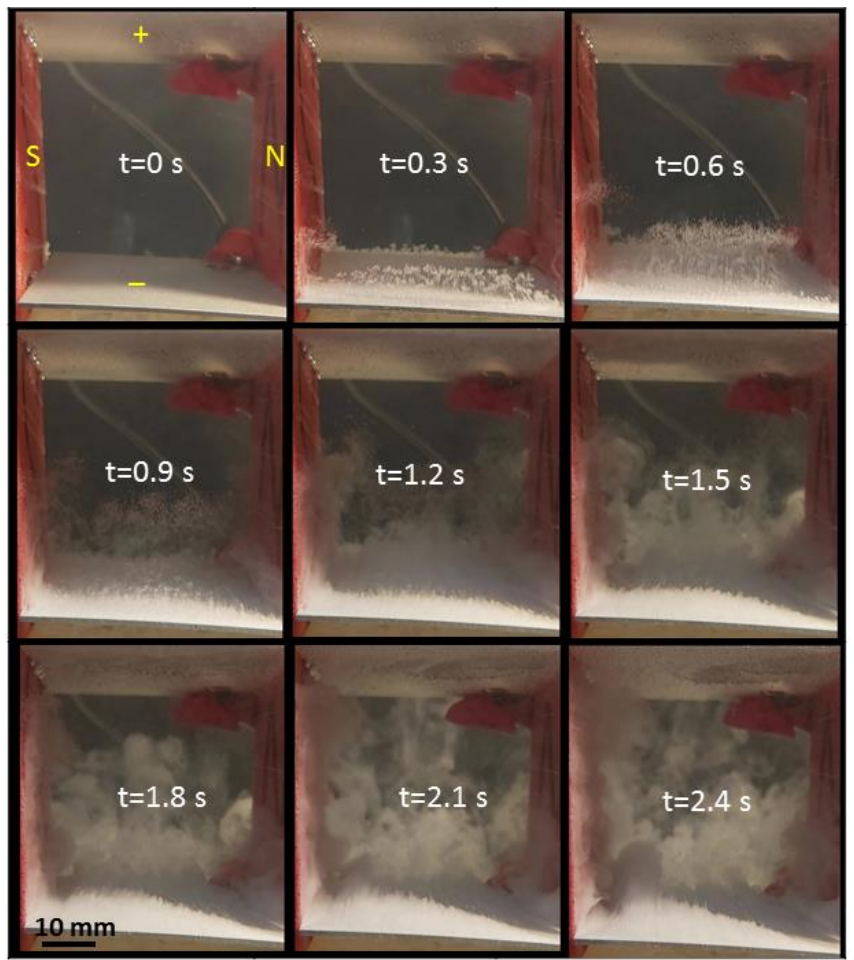

a

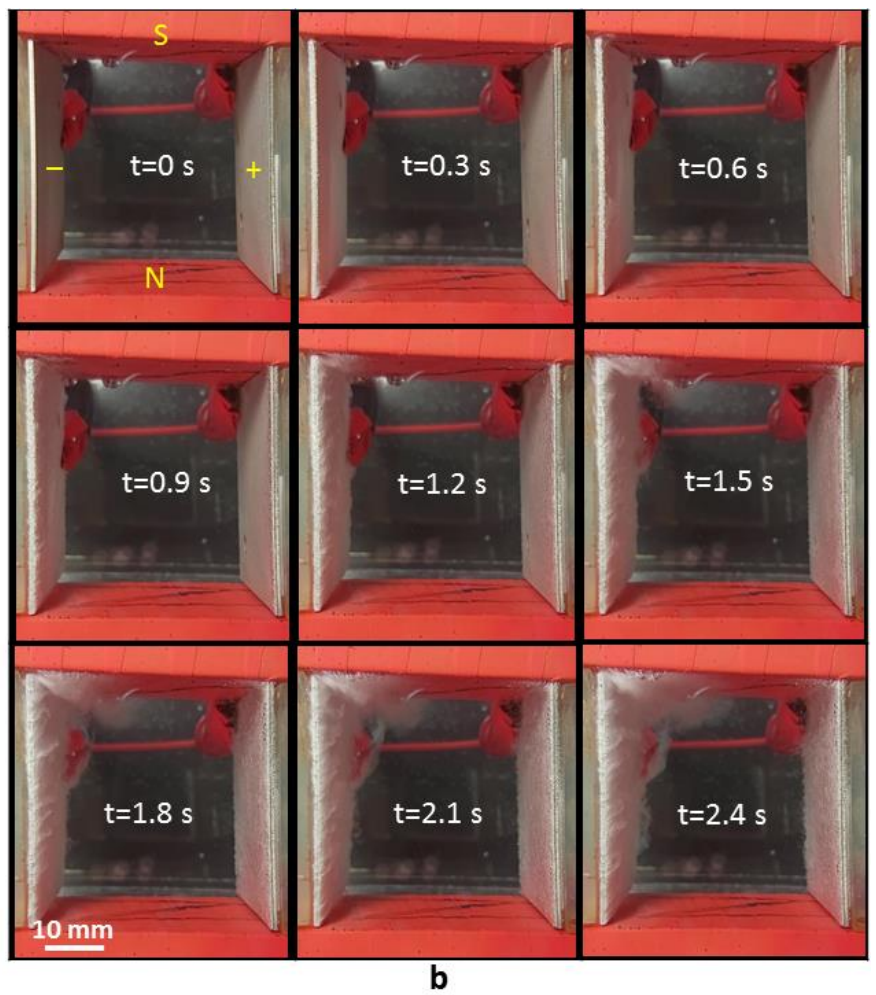

b

Figure 5. Locomotion of gas bubbles under the effect of horizontal Lorentz force with the different experimental layouts. (a) Sequential images of the oxygen and hydrogen bubbles detached from the upper and lower platinum electrode, respectively. The experimental layout is shown in figure 1c. The N-pole and S-pole of the magnet are at the right and left sides, respectively, under the electrical current of 5 A. (b) Sequential images of the oxygen and hydrogen bubbles detached from the right and left platinum electrode, respectively. The experimental layout is shown in figure 1f. The N-pole and S-pole of the magnet are at the lower and upper side, respectively. 
a
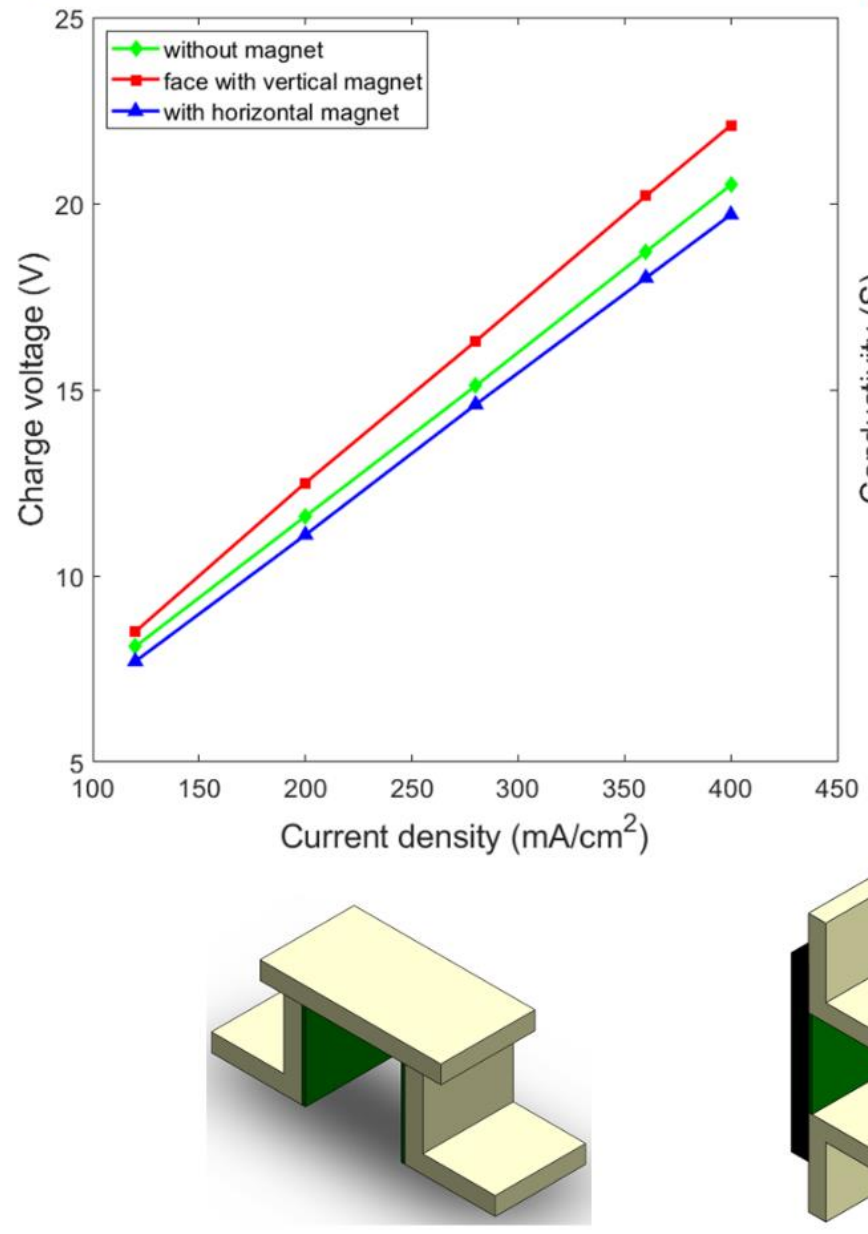

without magnet b

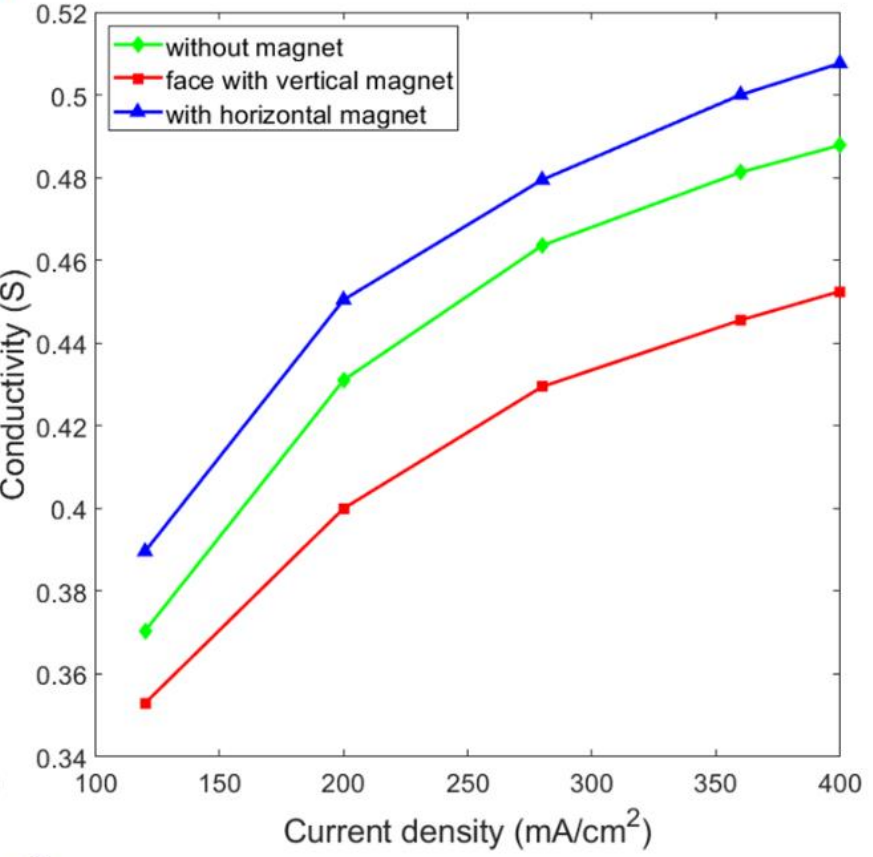

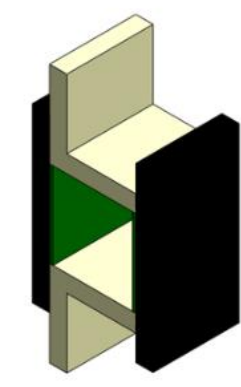

face with vertical magnet

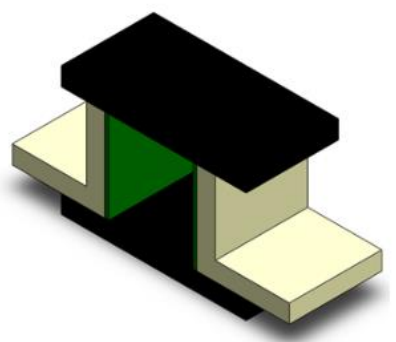

with horizontal magnet

Figure 6. Comparison of the conductivity for vertical platinum electrodes under the effects of various magnetic field configurations. (a) Charging voltage vs. current density for the experimental layouts shown in figures $1 \mathrm{~d}, 1 \mathrm{e}$, and 1f. (b) Conductivity vs. current density for the experimental layouts shown in figures $1 \mathrm{~d}, 1 \mathrm{e}$, and $1 \mathrm{f}$. 


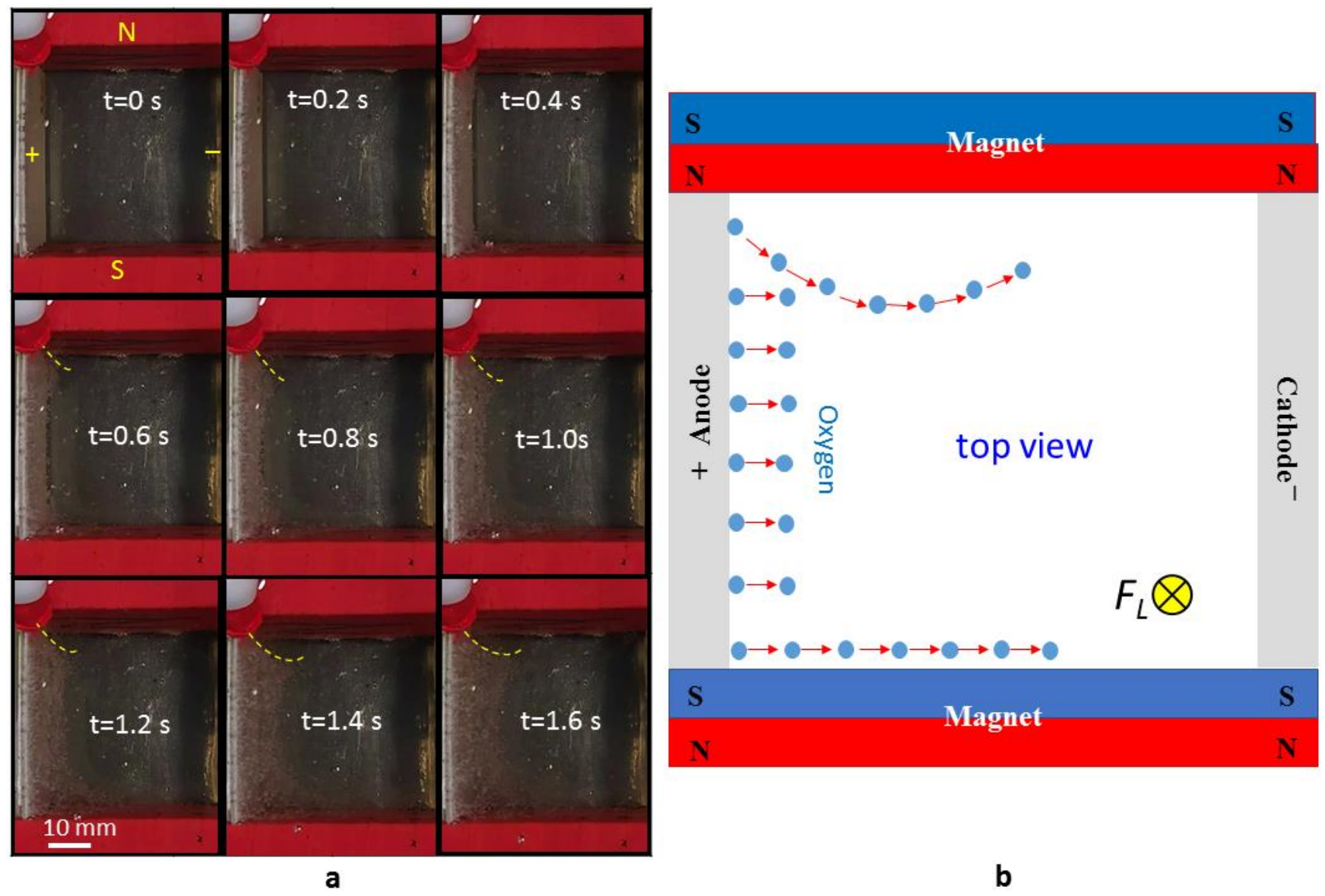

Figure 7. Repulsion of gas bubbles under the effect of the magnetic field parallel to the surface of the electrode. (a)Sequential images of the oxygen bubbles which are detached from the left platinum electrode and repelled and attracted by the N-pole and S-pole of the magnet, respectively. The experimental configuration is shown in figure 1(d), under an electrical current of 5A. (b) The schematic diagram for the locomotion and repulsion of the oxygen bubbles. 
a

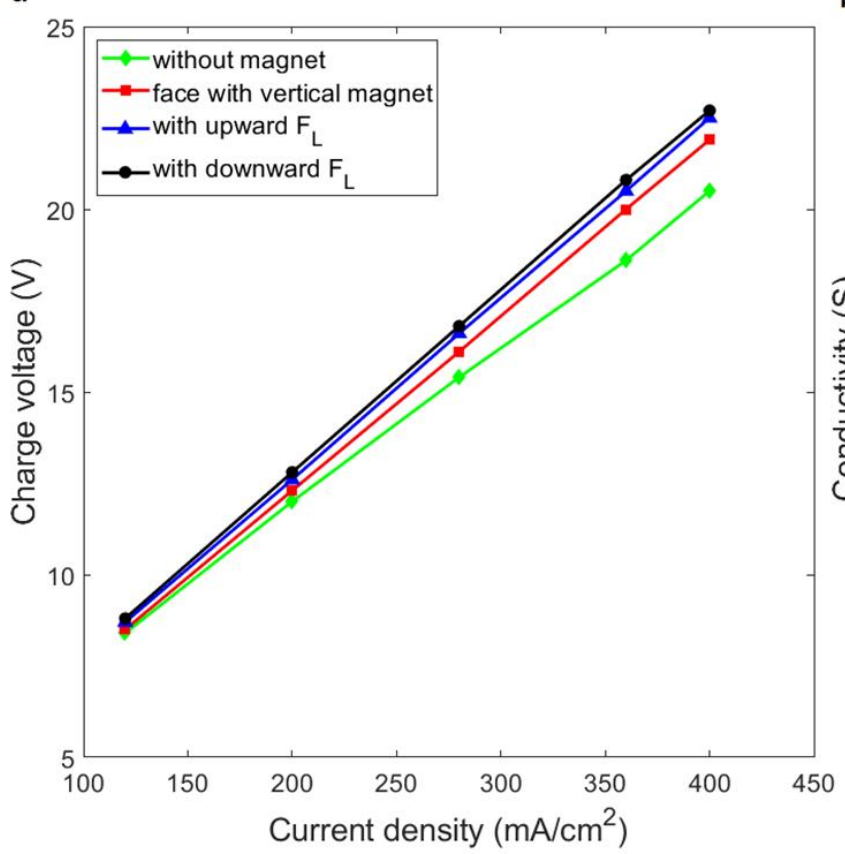

b

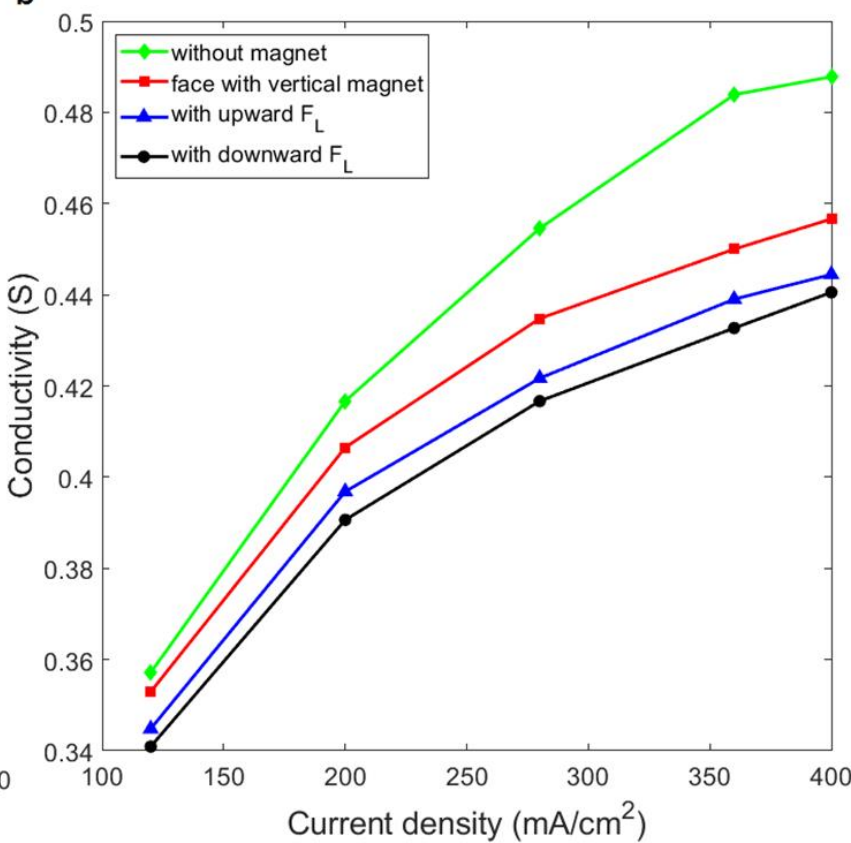

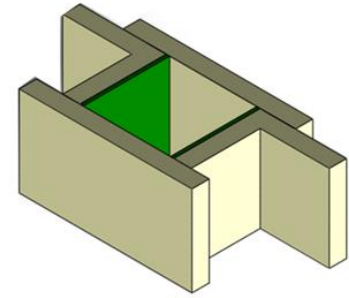

without magnet

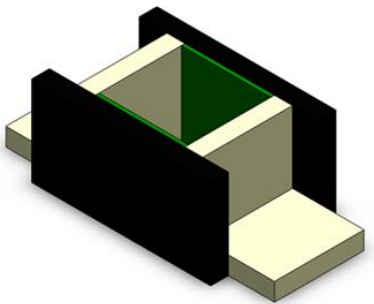

face with vertical magnet

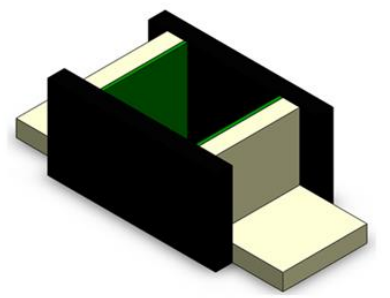

with vertical $\mathbf{F}_{\mathbf{L}}$

Figure 8. Comparison of the conductivity for vertical platinum electrodes under the effects of various magnetic field configurations which constitute a duct with an upward exit. (a) Charging voltage vs. current density for the experimental layout shown in figures $1 \mathrm{~d}, 1 \mathrm{e}$, and 1f. (b) Conductivity vs. current density for the experimental layout shown in figures $1 \mathrm{~d}, 1 \mathrm{e}$, and $1 \mathrm{f}$. 


\section{Figures}

a

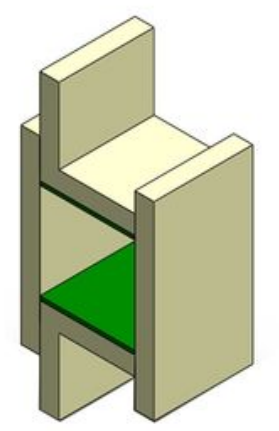

d

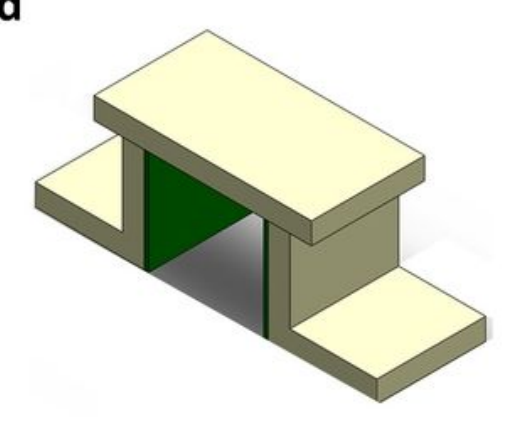

e

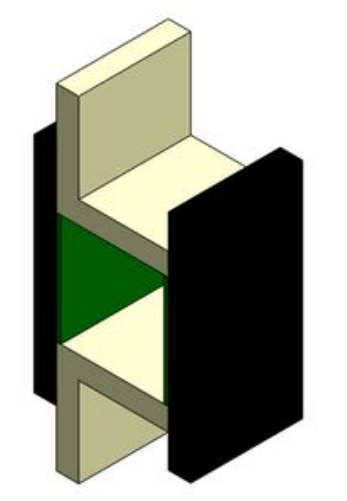

b

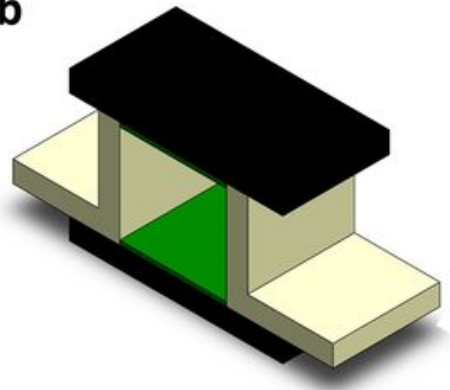

C

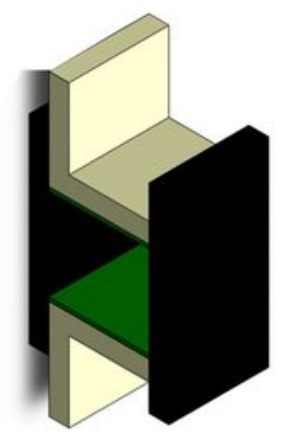

f
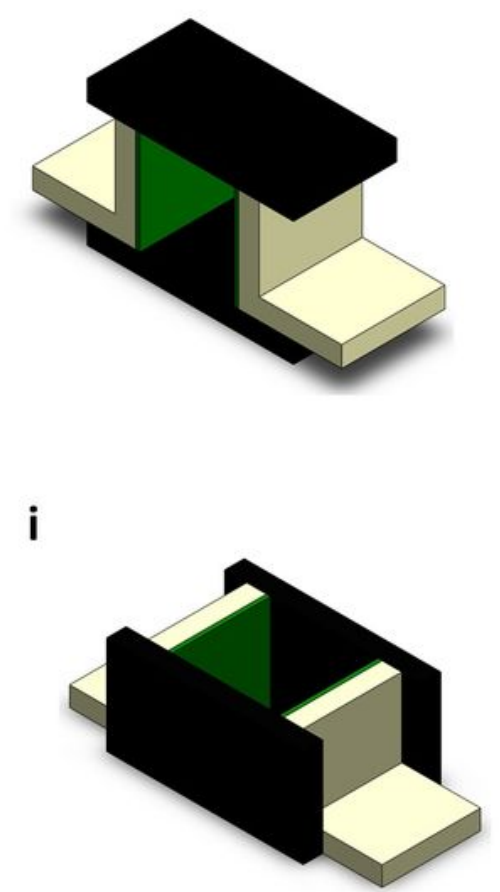

g

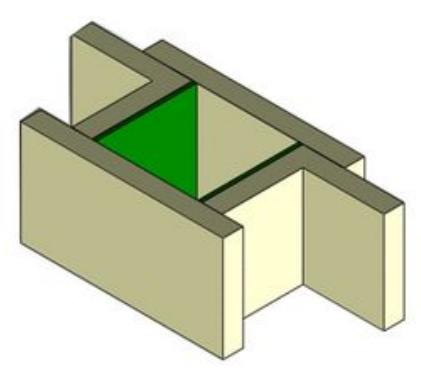

h

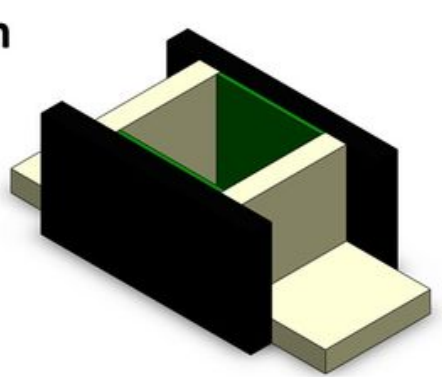

i

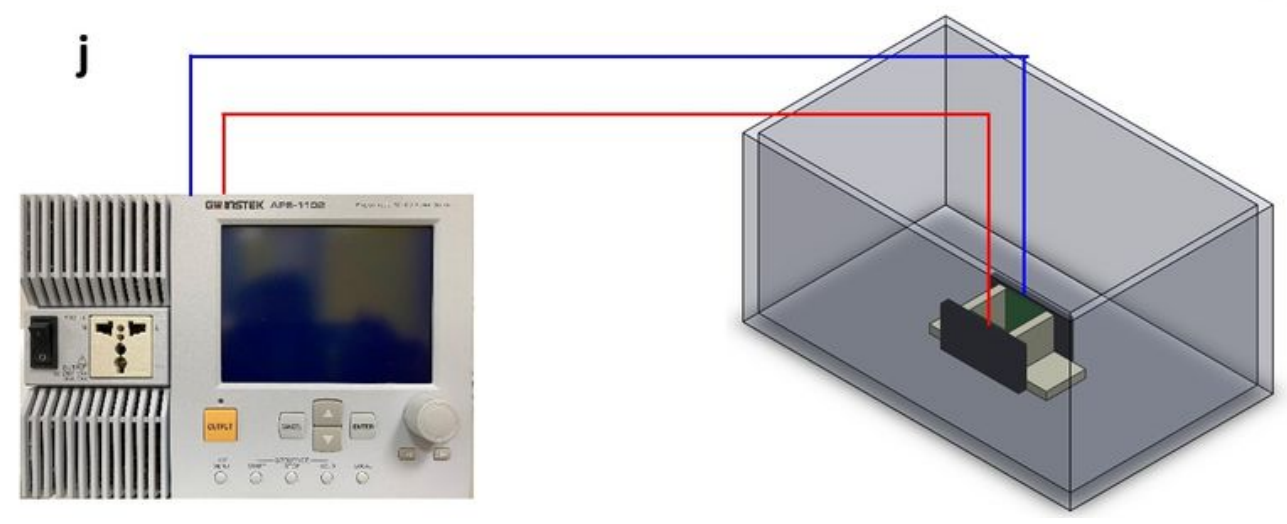

Figure 1

A schematic diagram of the experimental setup and the layout of the electrodes and magnetic field. The black area is the pair of N35 magnets (100 mm long, $50 \mathrm{~mm}$ wide, and $10 \mathrm{~mm}$ high) whose surface field strength is nearly $0.22 \mathrm{~T}$, the green part is the electrodes ( $50 \mathrm{~mm}$ long and $50 \mathrm{~mm}$ wide), the yellow part is 
the isolating plastic that is used to form the duct. Various magnetic field configurations were designed to investigate the effect of the field on the behaviors of the gas bubbles. (a) The electrodes were placed horizontally without magnets. (b) The electrodes were placed horizontally and faced with two horizontal magnets. (c) The electrodes were placed horizontally and perpendicular to the magnets which forms the Lorentz force (denoted as FL) in a horizontal direction. (d) The electrodes were placed vertically without magnet. (e) The electrodes were placed vertically and faced with vertical magnets. $(f)$ The electrodes were placed vertically and perpendicular to magnets which forms the Lorentz force in horizontal direction. (g) The electrodes were placed vertically without a magnet and with an upward exit. (h) The electrodes were placed vertically and faced with vertical magnets and with an upward exit. (i) The electrodes were placed vertically and perpendicular to magnets which forms the Lorentz force in a vertical direction and with an upward exit. (j) All the experiments were conducted in a $40 \mathrm{~cm}$ long, $24 \mathrm{~cm}$ wide, and $20 \mathrm{~cm}$ high water tank. The electrodes were connected to the power supply (GWInstek APS-1102) which can provide the maximum current of $10 \mathrm{~A}$.
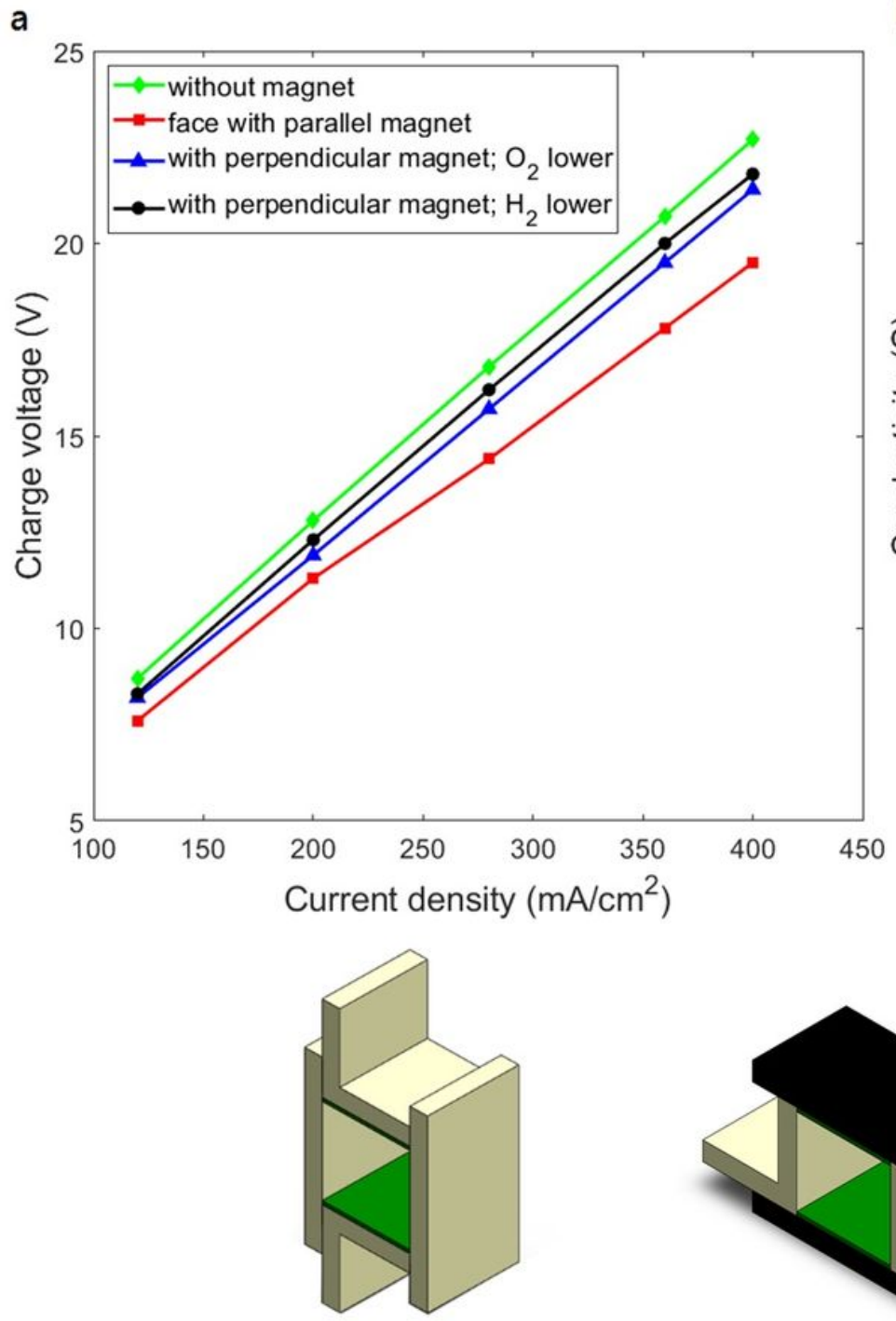

without magnet

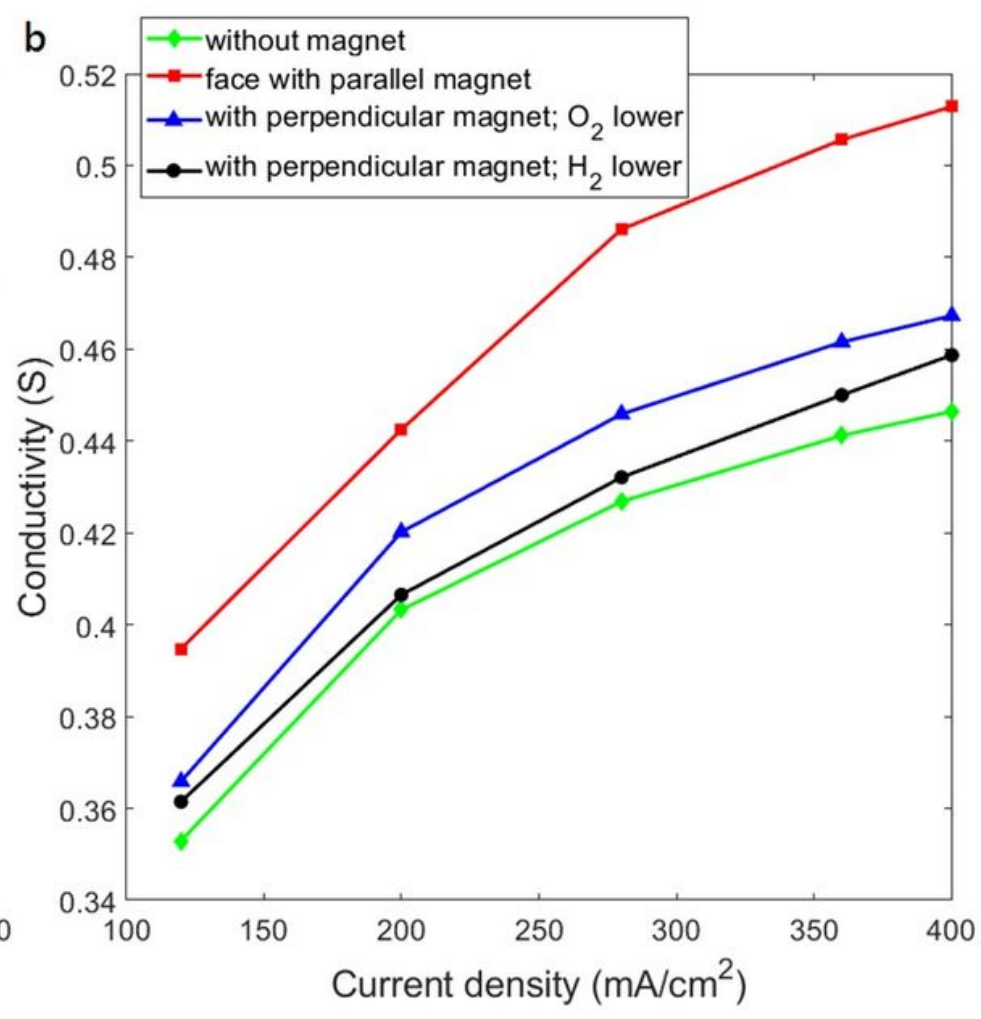

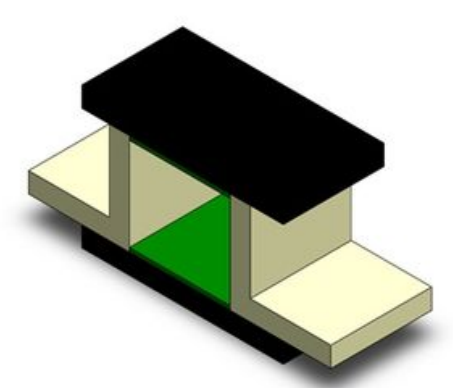

face with parallel magnet

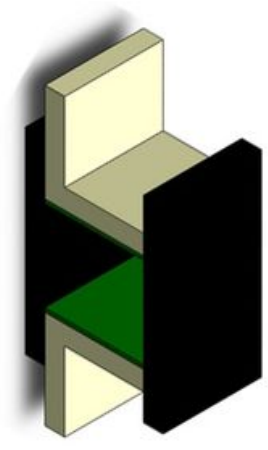

with perpendicular magnet

Figure 2 
Comparison of the conductivity for horizontal platinum electrodes under the effects of various magnetic field configurations. (a) Charging voltage vs. current density for the experimental layouts shown in figures $1 \mathrm{a}, 1 \mathrm{~b}$, and 1c. (b) Conductivity vs. current density for the experimental layouts shown in figures 1a, 1b, and $1 \mathrm{c}$.

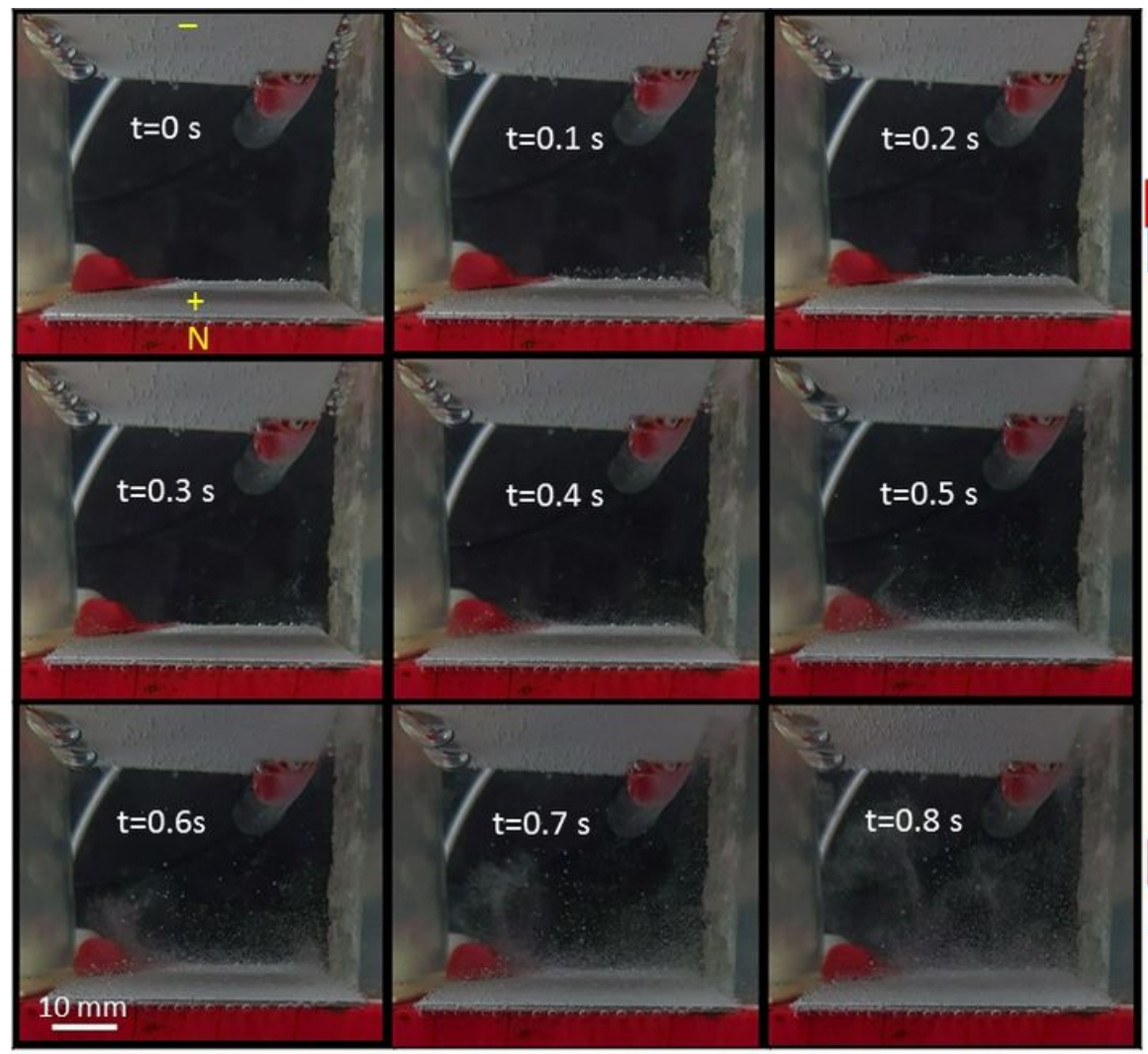

a

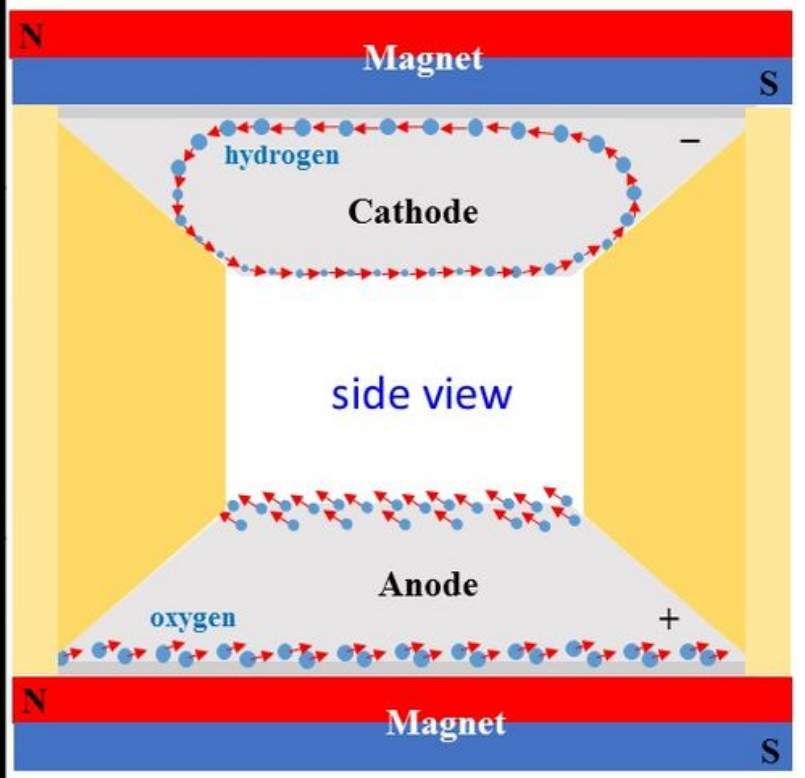

b

\section{Figure 3}

Swirling gas bubbles under the effect of the magnetic field perpendicular to the electrode surface. (a) Sequential images of the locomotion of the oxygen (lower electrode) and hydrogen (upper electrode) on the platinum electrodes subjected to a perpendicular magnetic field. The experimental layout is shown in figure 1(b) with an electrical current of $5 \mathrm{~A}$. (b) The schematic diagram for the revolution of the oxygen and hydrogen bubbles. 


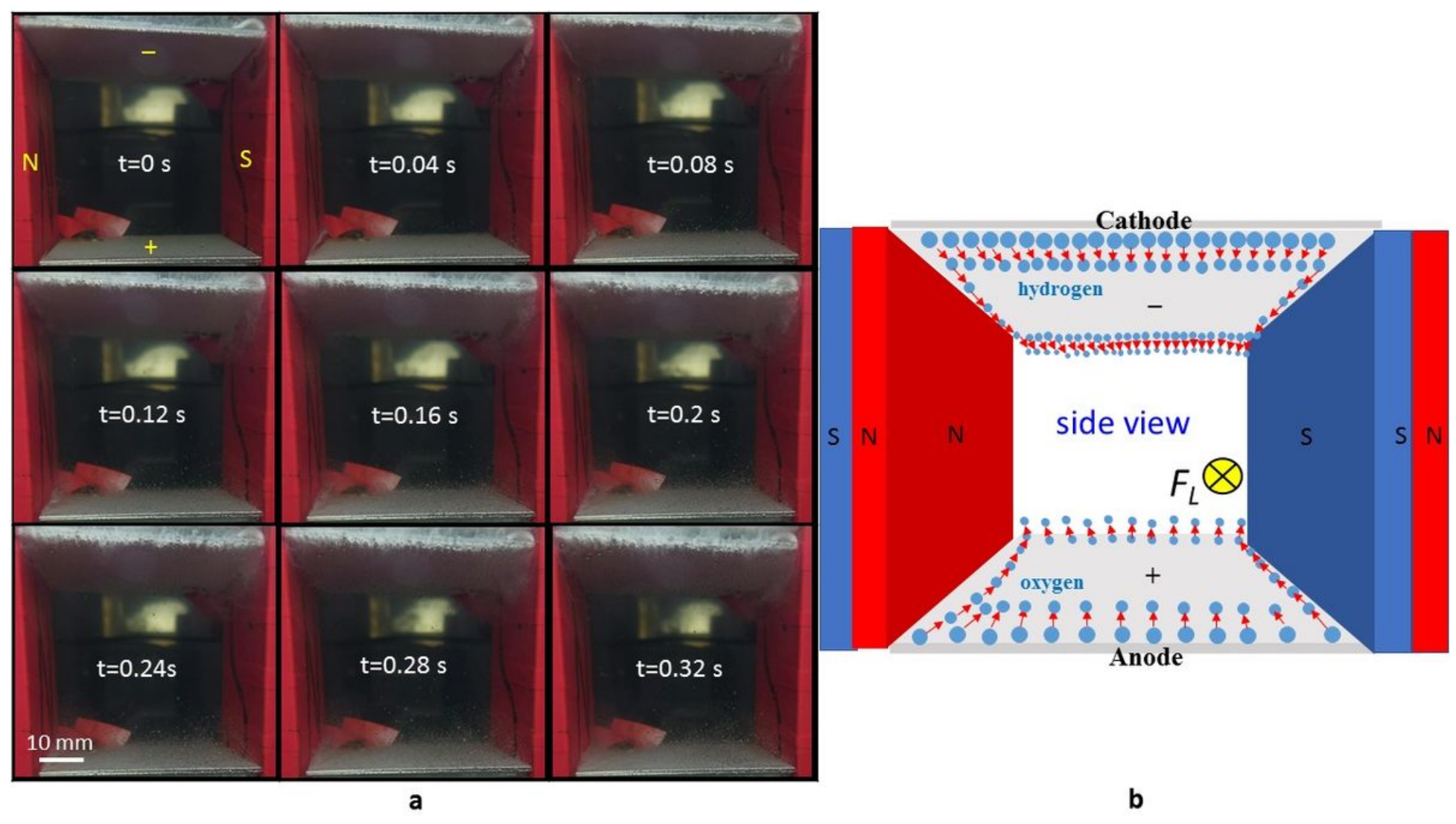

\section{Figure 4}

Locomotion of gas bubbles produced by horizontal platinum electrodes under the effect of horizontal Lorentz force generated by a horizontal magnetic field and a perpendicular electric field. (a)Sequential images of the oxygen and hydrogen bubbles detached from the lower and upper platinum electrode, respectively. The experimental layout is shown in figure 1 (c) under the electrical current of $5 \mathrm{~A}$. The N-pole and S-pole of the magnet are at the left and right sides, respectively. (b) The schematic diagram for the locomotion of the gas bubbles. 


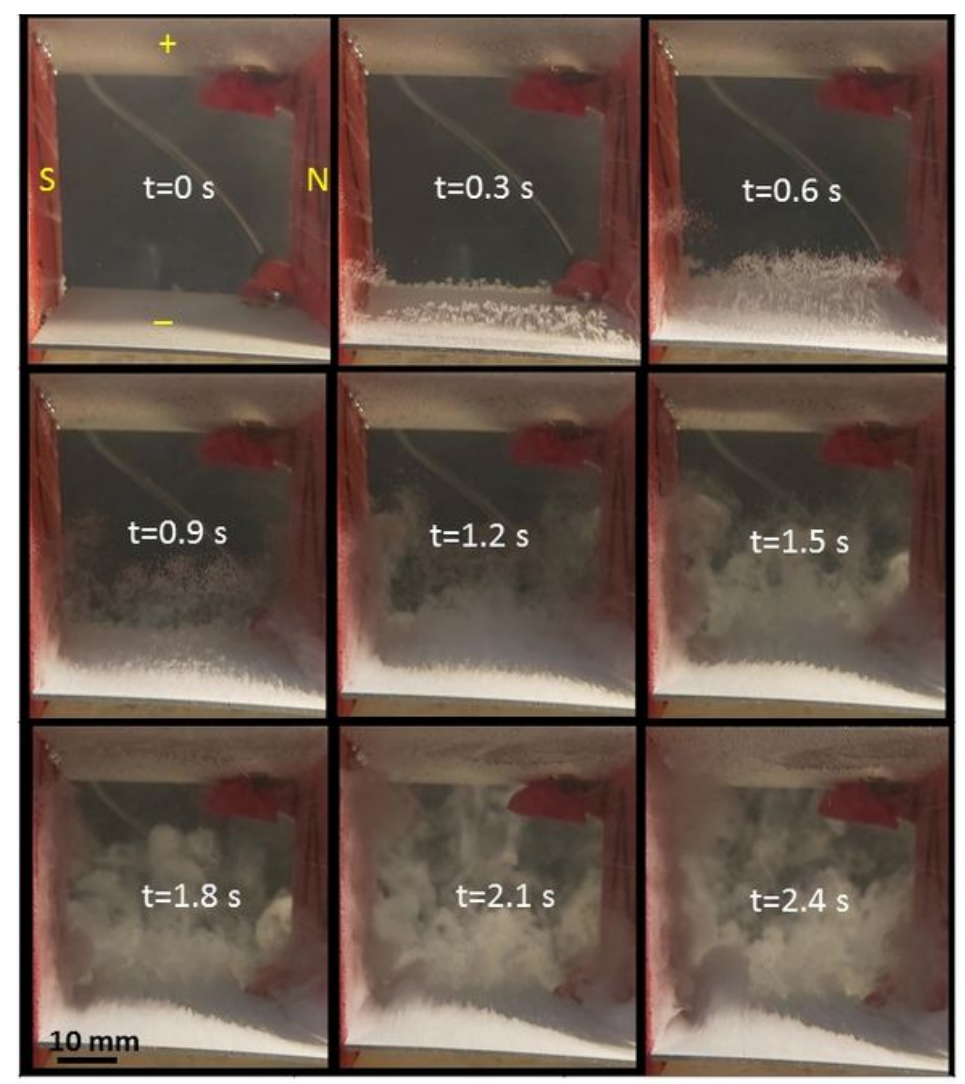

a

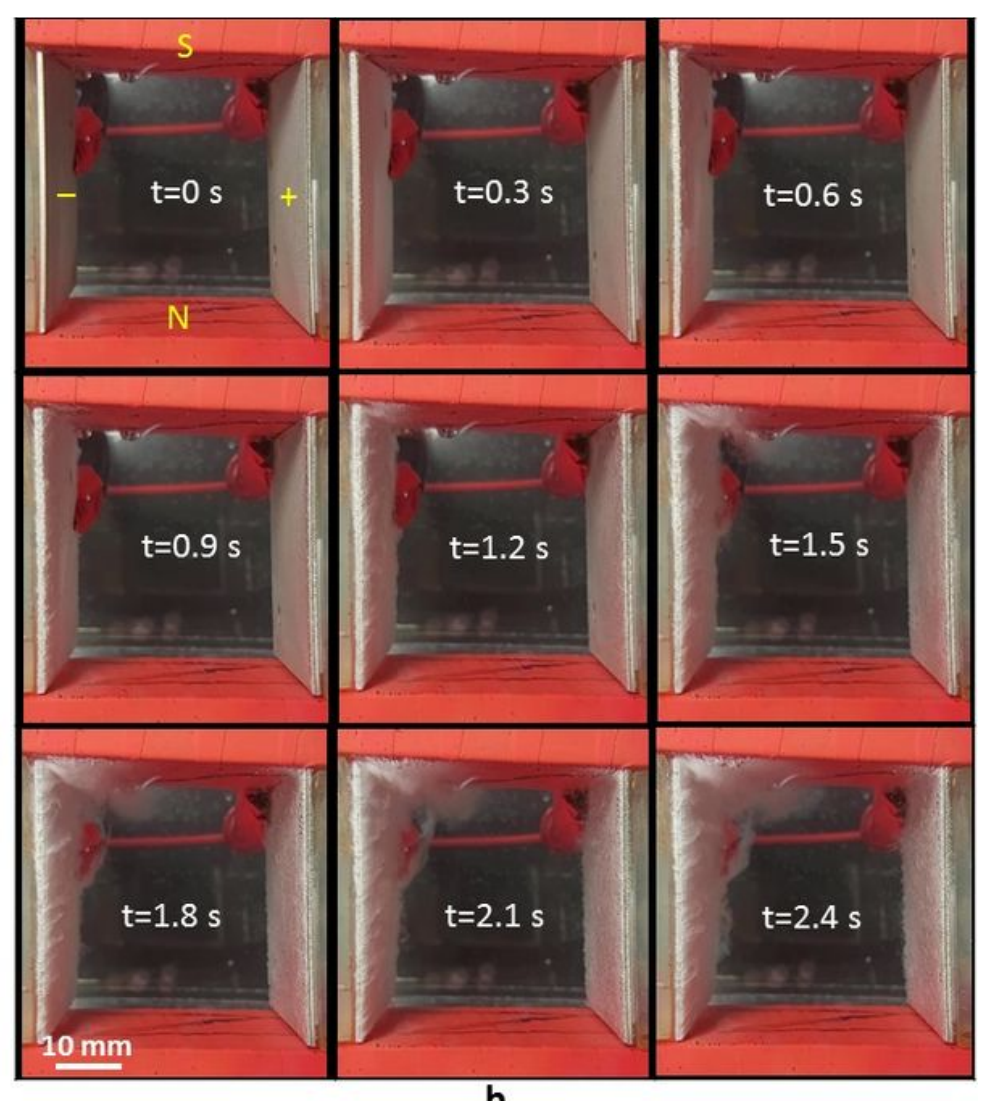

b

\section{Figure 5}

Locomotion of gas bubbles under the effect of horizontal Lorentz force with the different experimental layouts. (a) Sequential images of the oxygen and hydrogen bubbles detached from the upper and lower platinum electrode, respectively. The experimental layout is shown in figure 1c. The N-pole and S-pole of the magnet are at the right and left sides, respectively, under the electrical current of $5 \mathrm{~A}$. (b) Sequential images of the oxygen and hydrogen bubbles detached from the right and left platinum electrode, respectively. The experimental layout is shown in figure $1 \mathrm{f}$. The N-pole and S-pole of the magnet are at the lower and upper side, respectively. 
a
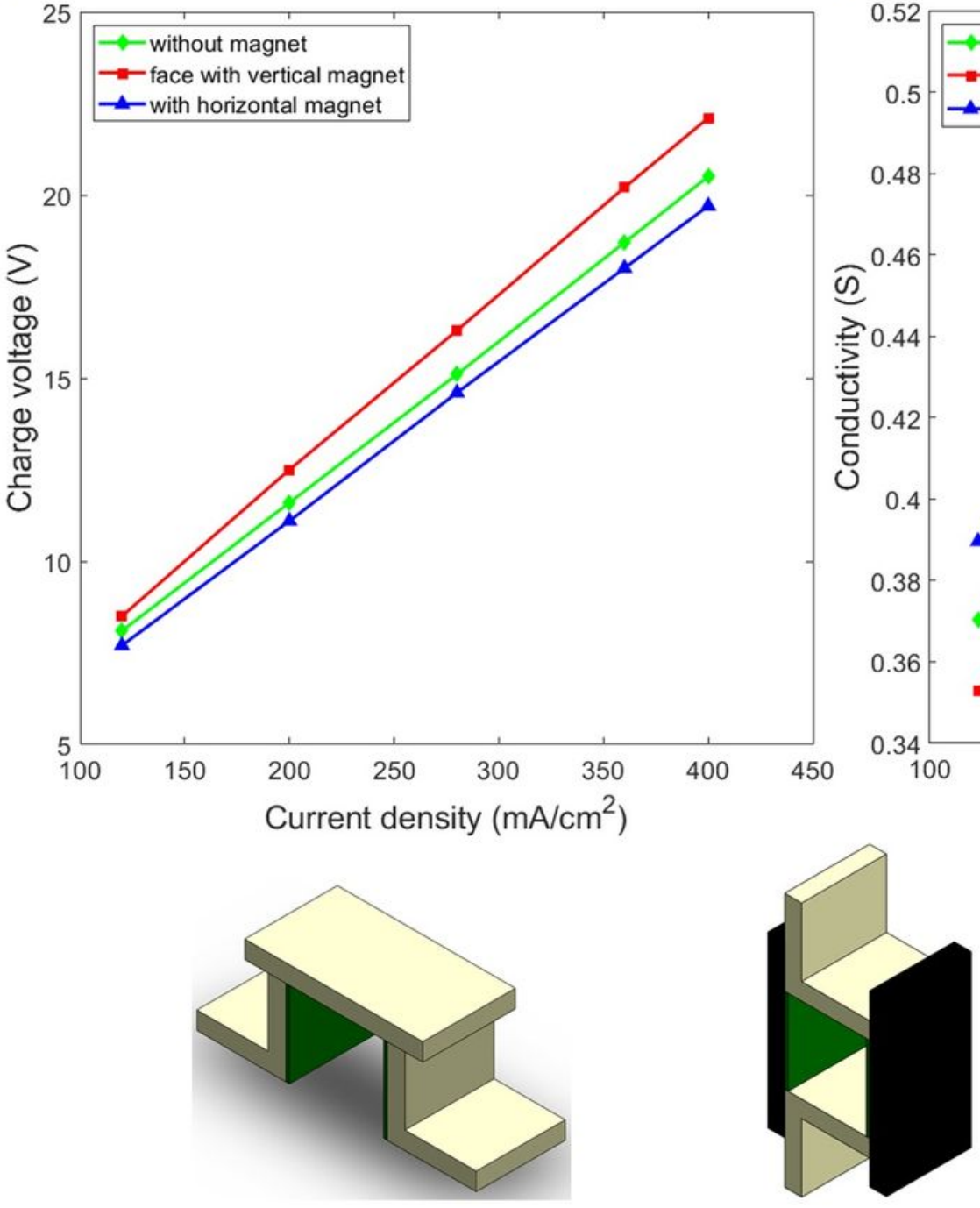

b without magnet

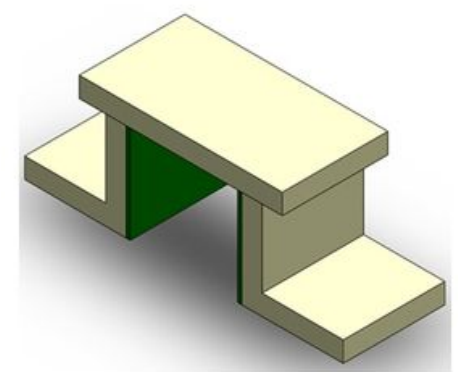

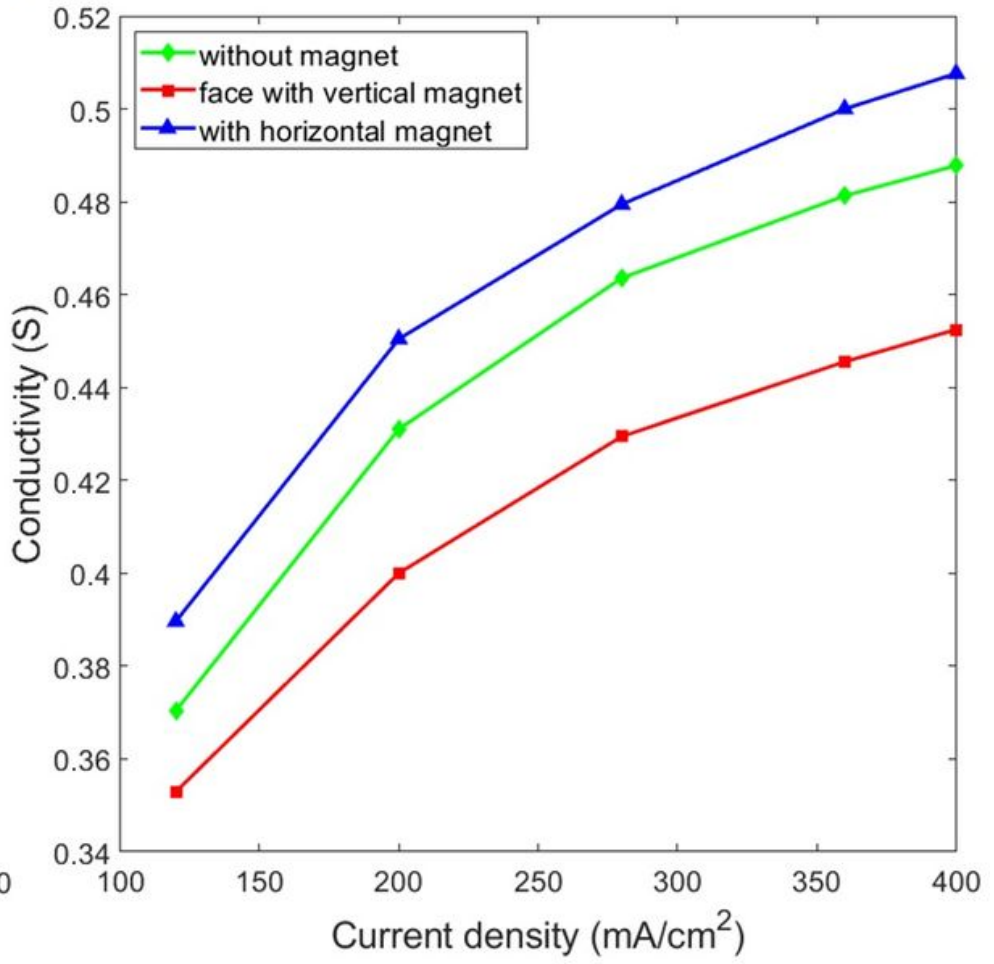

face with vertical magnet

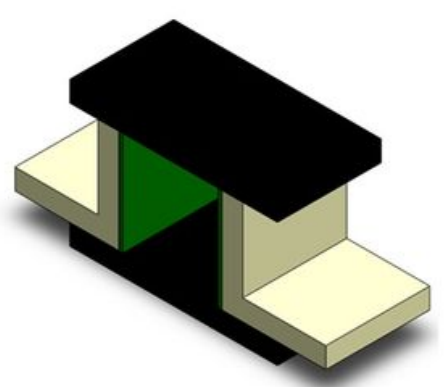

with horizontal magnet

\section{Figure 6}

Comparison of the conductivity for vertical platinum electrodes under the effects of various magnetic field configurations. (a) Charging voltage vs. current density for the experimental layouts shown in figures $1 \mathrm{~d}, 1 \mathrm{e}$, and 1f. (b) Conductivity vs. current density for the experimental layouts shown in figures 1d, 1e, and $1 f$. 


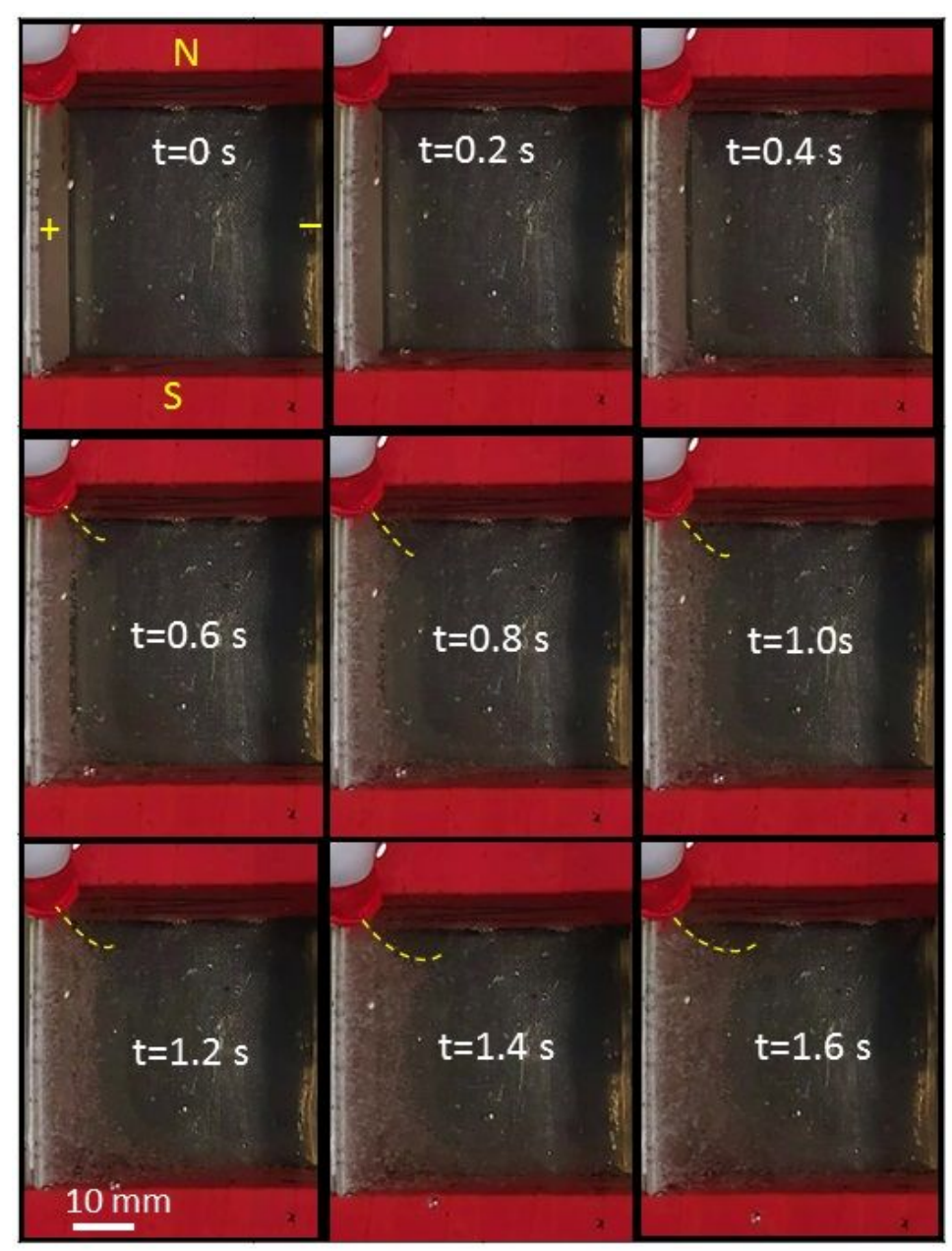

a

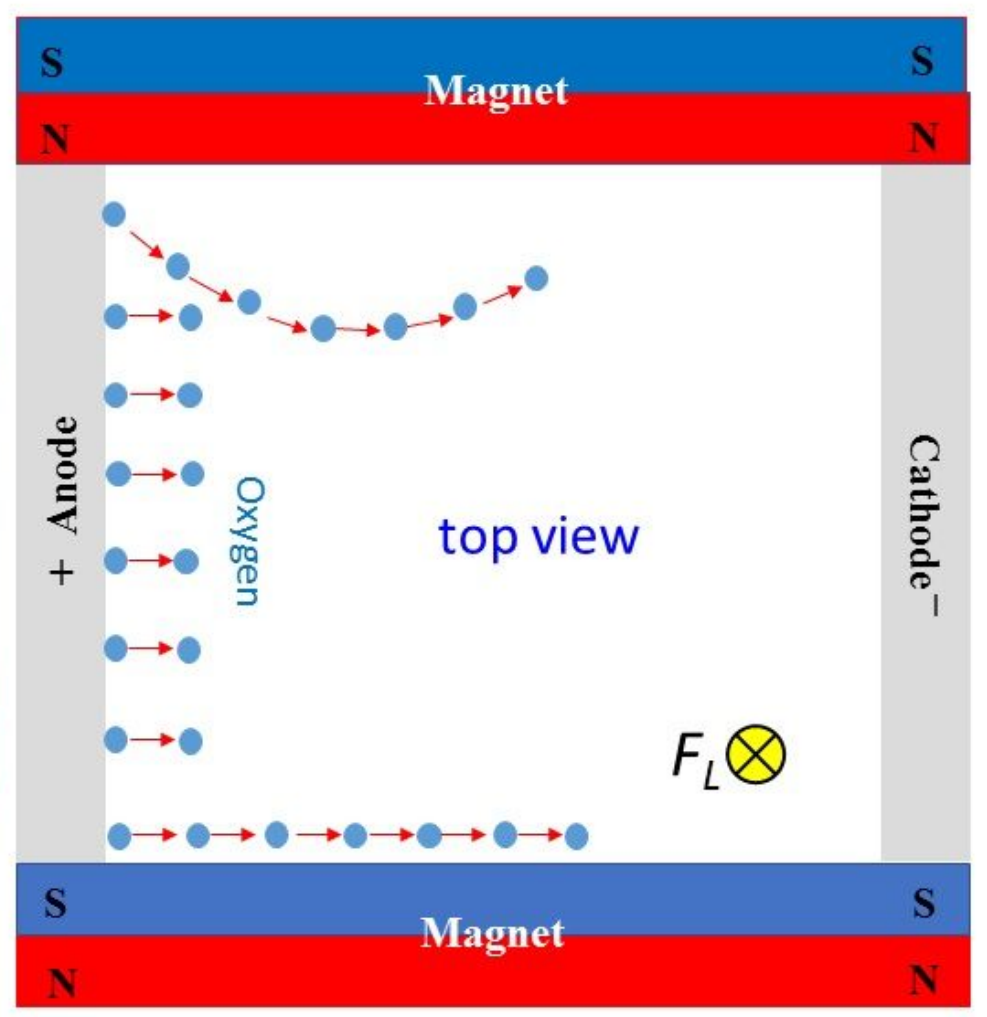

b

\section{Figure 7}

Repulsion of gas bubbles under the effect of the magnetic field parallel to the surface of the electrode. (a)Sequential images of the oxygen bubbles which are detached from the left platinum electrode and repelled and attracted by the $\mathrm{N}$-pole and S-pole of the magnet, respectively. The experimental configuration is shown in figure 1 (d), under an electrical current of $5 \mathrm{~A}$. (b) The schematic diagram for the locomotion and repulsion of the oxygen bubbles. 
a

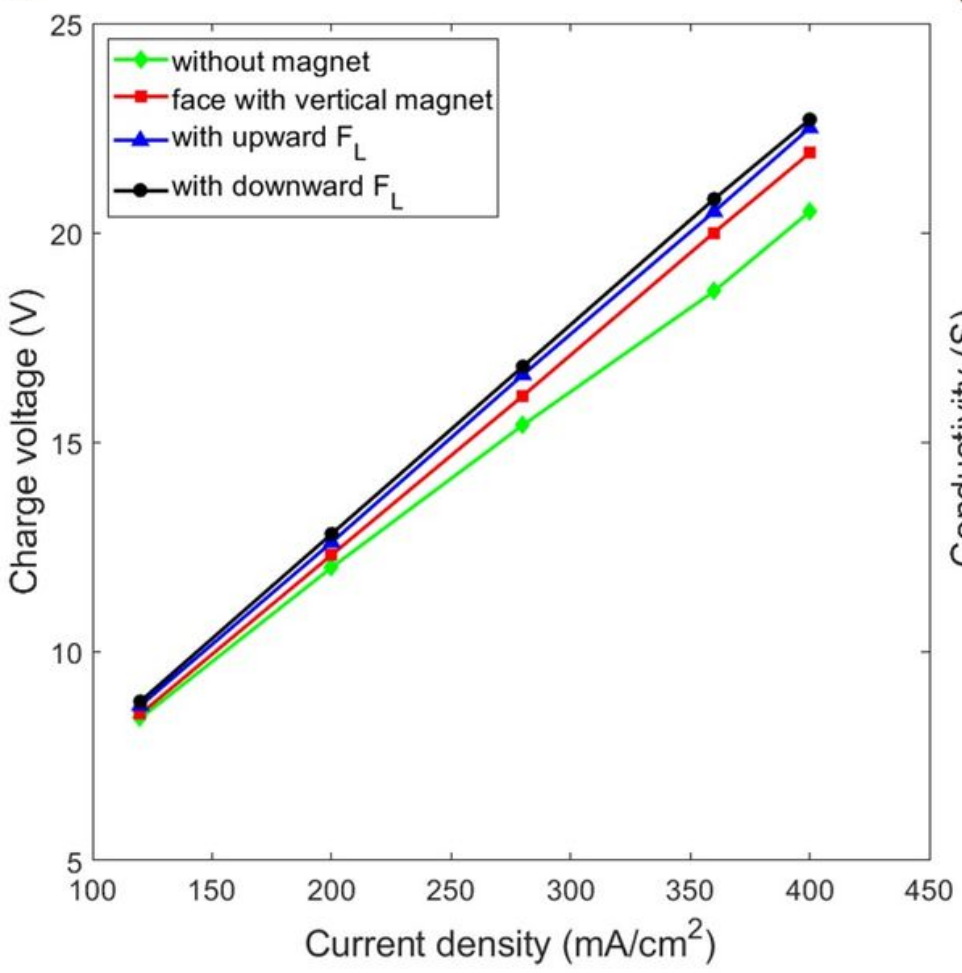

b

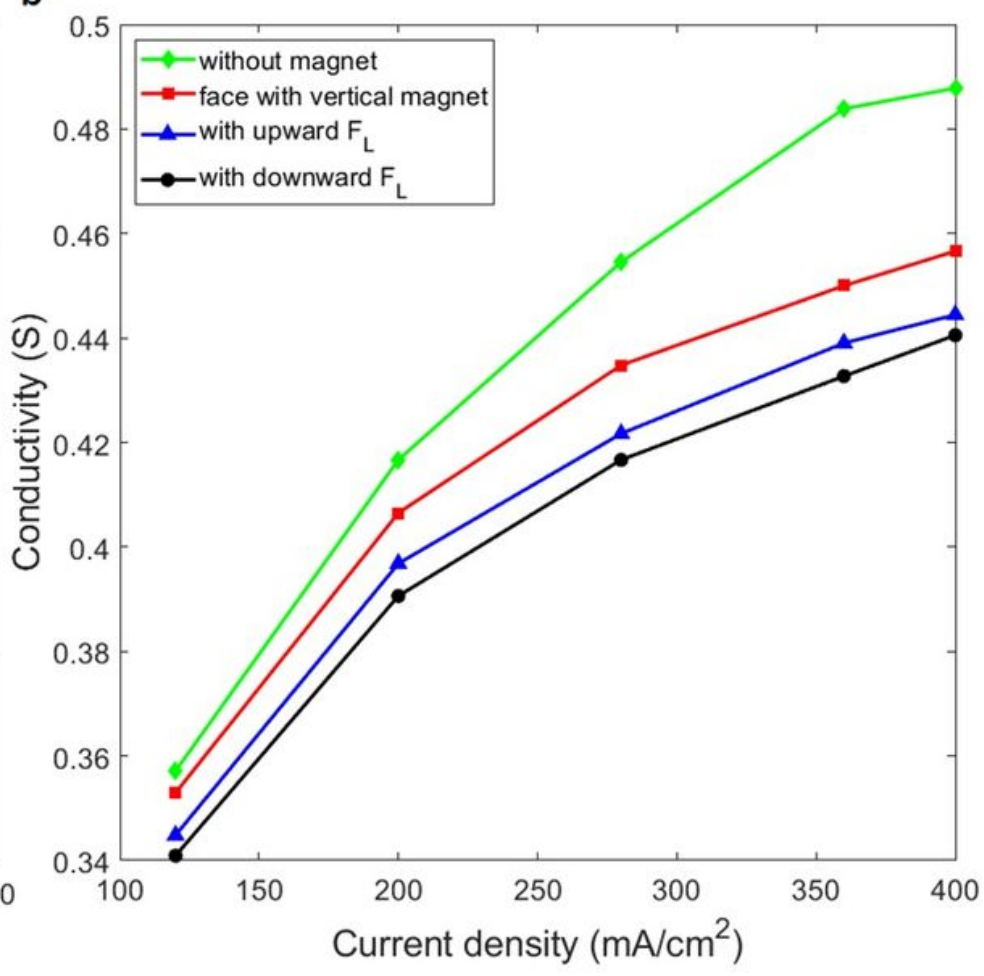

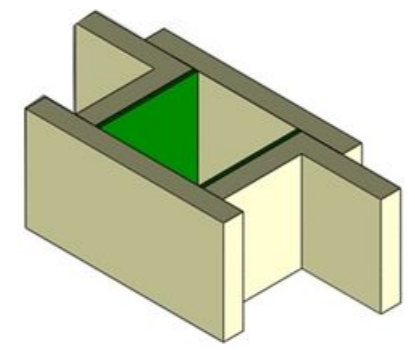

without magnet

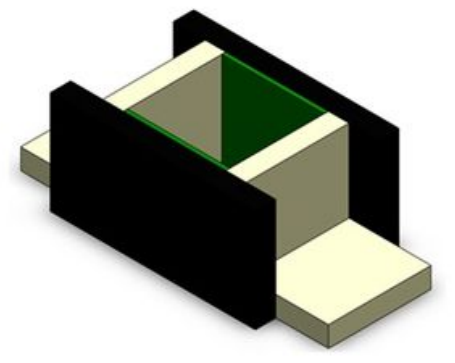

face with vertical magnet

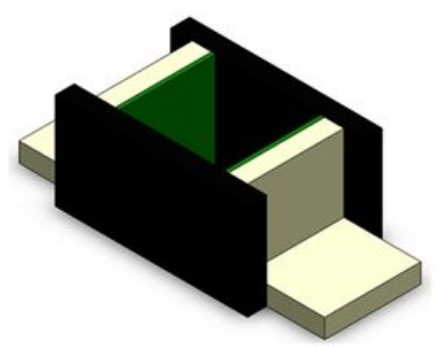

with vertical $F_{L}$

\section{Figure 8}

Comparison of the conductivity for vertical platinum electrodes under the effects of various magnetic field configurations which constitute a duct with an upward exit. (a) Charging voltage vs. current density for the experimental layout shown in figures $1 \mathrm{~d}, 1 \mathrm{e}$, and $1 \mathrm{f}$. (b) Conductivity vs. current density for the experimental layout shown in figures $1 \mathrm{~d}, 1 \mathrm{e}$, and $1 \mathrm{f}$.

\section{Supplementary Files}

This is a list of supplementary files associated with this preprint. Click to download.

- Googledrivelinkfortheexperimentalmovies.pdf 